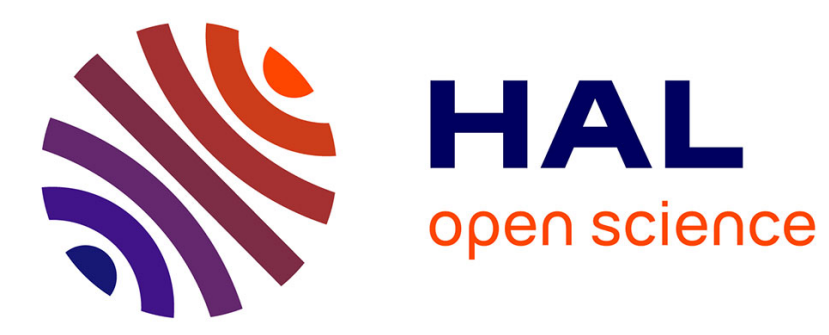

\title{
AN ANALYSIS OF HIGHER ORDER BOUNDARY CONDITIONS FOR THE WAVE EQUATION
}

\author{
Julien Diaz, Patrick Joly
}

\section{To cite this version:}

Julien Diaz, Patrick Joly. AN ANALYSIS OF HIGHER ORDER BOUNDARY CONDITIONS FOR THE WAVE EQUATION. SIAM Journal on Applied Mathematics, 2005, 65 (5), pp.1547-1575. $10.1137 /$ S0036139903436145 . inria-00409197

\section{HAL Id: inria-00409197 https://hal.inria.fr/inria-00409197}

Submitted on 6 Aug 2009

HAL is a multi-disciplinary open access archive for the deposit and dissemination of scientific research documents, whether they are published or not. The documents may come from teaching and research institutions in France or abroad, or from public or private research centers.
L'archive ouverte pluridisciplinaire HAL, est destinée au dépôt et à la diffusion de documents scientifiques de niveau recherche, publiés ou non, émanant des établissements d'enseignement et de recherche français ou étrangers, des laboratoires publics ou privés. 


\title{
AN ANALYSIS OF HIGHER ORDER BOUNDARY CONDITIONS FOR THE WAVE EQUATION*
}

\author{
JULIEN DIAZ ${ }^{\dagger}$ AND PATRICK JOLY ${ }^{\dagger}$
}

\begin{abstract}
Thanks to the use of the Cagniard-De Hoop method, we derive an analytic solution in the time domain for the half-space problem associated with the wave equation with EngquistMajda higher order boundary conditions. This permits us to derive new convergence results when the order of the boundary condition tends to $+\infty$, as well as error estimates. The theory is illustrated by numerical results.
\end{abstract}

Key words. Cagniard-De Hoop method, absorbing boundary conditions, wave equation, error estimates, convergence, Green's function

AMS subject classifications. 35L05, 35L20, 35C05, 65M99, 44E99

DOI. $10.1137 /$ S0036139903436145

1. Introduction. The design of accurate absorbing boundary conditions (ABCs) for the numerical calculation of waves in the time domain is already an old subject since the major work of Engquist and Majda [11], [12] in the late 1970s. Their main contribution was the construction and analysis of a hierarchy of local boundary conditions for the wave equation. Let us concentrate on the two-dimensional (2D) acoustic wave equation:

$$
\frac{1}{c^{2}} \frac{\partial^{2} u}{\partial t^{2}}-\Delta u=0, \quad x=\left(x_{1}, x_{2}\right) \in \mathbb{R}^{2}, \quad t>0 .
$$

Assuming that the data (initial data) of the problem are supported in the upper half-space $\mathbb{R}_{+}^{2}=\left\{x_{2}>0\right\}$, it is natural to try to reduce the effective numerical computations to this half-space by imposing adequate absorbing boundary conditions on the artificial boundary $\Gamma=\partial \mathbb{R}_{+}^{2}$. In [11], Engquist and Majda proposed the following condition (the integer $N$ is a parameter meant to be large):

$$
\mathcal{B}^{N} u=0 \quad \text { on } \Gamma,
$$

where the operators

$$
\mathcal{B}^{N}=\mathcal{B}^{N}\left(\frac{\partial}{\partial t}, \frac{\partial}{\partial x_{1}}, \frac{\partial}{\partial x_{2}}\right), \quad N \geq 0,
$$

are a family of homogeneous differential operators defined inductively by

$$
\left\{\begin{array}{l}
\mathcal{B}^{0}=2 I, \quad \mathcal{B}^{1}=\frac{\partial}{\partial t}-c \frac{\partial}{\partial x_{2}} \\
\mathcal{B}^{N+1}=\frac{\partial}{\partial t} \mathcal{B}^{N}-\frac{c^{2}}{4} \frac{\partial^{2}}{\partial x_{1}^{2}} \mathcal{B}^{N-1} .
\end{array}\right.
$$

\footnotetext{
${ }^{*}$ Received by the editors October 14, 2003; accepted for publication (in revised form) October 18, 2004; published electronically May 12, 2005.

http://www.siam.org/journals/siap/65-5/43614.html

†INRIA Rocquencourt, Domaine de Voluceau, B. P. 105, 78153 Le Chesnay, France (julien.diaz@ inria.fr, patrick.joly@inria.fr).
} 
Note that $\mathcal{B}^{N}$ can be rewritten in the form

$$
\mathcal{B}^{N}=S_{N-1}\left(\frac{\partial}{\partial t}, \frac{\partial}{\partial x_{1}}\right) \frac{\partial}{\partial x_{2}}-Q_{N}\left(\frac{\partial}{\partial t}, \frac{\partial}{\partial x_{1}}\right),
$$

where $Q_{N}$ and $S_{N-1}$ are homogeneous polynomials of two variables of respective degrees $N$ and $N-1$. In particular, $\mathcal{B}^{N}$ remains of first order with respect to $x_{2}$; the condition (1.2) can be seen as a Dirichlet-to-Neumann (or impedance) type boundary condition since it can be formally rewritten as

$$
\frac{\partial u}{\partial x_{2}}-\frac{Q_{N}\left(\frac{\partial}{\partial t}, \frac{\partial}{\partial x_{1}}\right)}{S_{N-1}\left(\frac{\partial}{\partial t}, \frac{\partial}{\partial x_{1}}\right)} u=0 .
$$

Remark 1.1. For smooth solutions (up to the boundary $\Gamma$ ) of the wave equation, the boundary condition (1.2) can be rewritten in terms of $t$ and $x_{2}$ derivatives only. Indeed, $\mathcal{B}^{N}$ is obviously even with respect to the $x_{1}$ variable, and, thanks to the wave equation, the second order derivative with respect to $x_{1}$ can be replaced by $t$ and $x_{2}$ derivatives:

$$
\frac{\partial^{2}}{\partial x_{1}^{2}} \longrightarrow \frac{1}{c^{2}} \frac{\partial^{2}}{\partial t^{2}}-\frac{\partial^{2}}{\partial x_{2}^{2}}
$$

As a consequence, one can show that [21]

$$
\mathcal{B}^{N} u=0 \Longleftrightarrow \widetilde{\mathcal{B}}^{N} u=0, \quad \widetilde{\mathcal{B}}^{N}=\left(\frac{\partial}{\partial t}-c \frac{\partial}{\partial x_{2}}\right)^{N} .
$$

This remark will be useful in section 3 .

Let us recall that the initial boundary value problems (IBVPs) for linear hyperbolic systems (1.2), or (1.4) or (1.5), are constructed as an approximation of an exact or transparent boundary condition

$$
\mathcal{B} u=0, \quad \mathcal{B}=\frac{\partial}{\partial x_{2}}-\mathcal{L},
$$

where $\mathcal{L}$ is a pseudodifferential operator in $\left(x_{1}, t\right)$ whose symbol is known explicitly. More precisely, if one uses the Laplace-Fourier transform in the $\left(t, x_{1}\right)$ plane (see (3.3) and $(3.2))$,

$$
\varphi\left(x_{1}, t\right) \rightarrow \widetilde{\varphi}(k, s)
$$

one has the formula

$$
\widetilde{\mathcal{L} \varphi}(k, s)=\left(k^{2}+\frac{s^{2}}{c^{2}}\right)^{\frac{1}{2}} \varphi(k, s), \quad \mathcal{R} e\left(k^{2}+\frac{s^{2}}{c^{2}}\right)^{\frac{1}{2}} \geq 0 .
$$

This comes from the fact that if $u$ is a solution of the wave equation in the lower halfspace $\mathbb{R}_{-}^{2}=\left\{x_{2}<0\right\}$ with zero initial data, its partial Laplace-Fourier transform in $t$ and $x_{1}, \widetilde{u}\left(k, x_{2}, s\right)$, satisfies

$$
\widetilde{u}\left(k, x_{2}, s\right)=\widetilde{u}(k, 0, s) e^{\left(k^{2}+\frac{s^{2}}{c^{2}}\right)^{\frac{1}{2}} x_{2}}, \quad x_{2} \leq 0,
$$


which yields in particular

$$
\frac{d \widetilde{u}}{d x_{2}}(k, 0, s)-\left(k^{2}+\frac{s^{2}}{c^{2}}\right)^{\frac{1}{2}} \widetilde{u}(k, 0, s)=0 .
$$

The presence of the square root in the symbol of $\mathcal{L}$ makes the operator $\mathcal{L}$, and consequently the boundary condition (1.6), nonlocal in space and time, which is a priori very unpleasant from the numerical point of view. The approximate condition simply comes from a rational approximation of the symbol of $\mathcal{L}$ in such a way that the resulting boundary condition can be expressed in terms of differential operators, which is much more tractable from the numerical point of view. If one writes

$$
\left(k^{2}+\frac{s^{2}}{c^{2}}\right)^{\frac{1}{2}}=\frac{s}{c}\left(1+\frac{c^{2} k^{2}}{s^{2}}\right)^{\frac{1}{2}},
$$

the problem is reduced to the rational approximation of the function of one variable:

$$
f(z)=\left(1+z^{2}\right)^{\frac{1}{2}} .
$$

Noticing that $f(z)$ is a solution of the fixed point equation,

$$
f(z)=1+\frac{z^{2}}{1+f(z)},
$$

one obtains a rational approximation (or continuous fraction expansion) of $f(z)$ with the following fixed point algorithm:

$$
f_{n+1}(z)=1+\frac{z^{2}}{1+f_{n}(z)}, \quad f_{1}(z)=1 .
$$

The condition $(1.2)$ is obtained by replacing in (1.6) $\mathcal{L}$ by $\mathcal{L}_{N}$, whose symbol is $\frac{s}{c} f_{N}\left(\frac{c k}{s}\right)$. It is then relatively easy to deduce the induction formula (1.3) from (1.9).

Remark 1.2. It is easy to show that the sequence $f_{n}(z)$ converges, for large $n$, to $f(z)$ only if $|z|<1$. Moreover, the convergence is uniform and exponential in any compact of the unit circle. For $|z|>1, f_{n}(z)$ converges to $-f(z)$, which is the other solution of (1.8). However, it is not a problem for the application to ABCs, as will be shown in this paper.

It is also well known that (1.9) provides the sequence of $\{n, n-1\}$ (for even $n$ ) and $\{n-1, n-1\}$ (for odd $n$ ) Padé approximants [3] of $f(z)$ at the neighborhood of the origin:

$$
f_{2}(z)=1+\frac{z^{2}}{2}, \quad f_{3}(z)=1+\frac{2 z^{2}}{4+z^{2}}, \ldots ;
$$

and in particular one has

$$
f_{n}(z)-f(z)=O\left(\left(z^{2}\right)^{N}\right), \quad z \rightarrow 0 .
$$

That is why the boundary condition (1.2) is known as the Engquist-Majda condition of order $2 \mathrm{~m}$. Equation (1.10) shows that the rational approximation of the symbol of $\mathcal{L}$ given by (1.9) is better for the small values of $c k / s$, which has a physical interpretation (see below).

During recent years, abundant research has been devoted to various improvements (including in particular "better" rational approximations) and extensions (including in 
particular the application to other wave equations) of the Engquist-Majda conditions. It is not possible to give here an exhaustive bibliography, and we will refer the reader to recent review papers on the subject by Hagström [18], [19] and Givoli [13]. In the last decade, alternative solutions have been progressively developed and, especially, researchers have tried to promote again the use of exact nonlocal boundary either by using specific geometries for the absorbing boundaries, as in the works by Grote and Keller [14], [15], or by exploiting the recent progress in rapid algorithms (multipoles) and rational approximation, as in the work of Alpert, Greengard, and Hagström [2], [1]. Approximately during the same period, the introduction by Berenger of the perfectly matched layers (PMLs) technique [6], [5] partly revolutionized the subject. The philosophy here is to replace the absorbing boundary with an absorbing layer (or sponge layer) which is such that any wave propagating in the computational domain is transmitted to the absorbing layer without being reflected. This method quickly attracted many researchers in different fields of application, in particular because of its good practical performances and its easy implementation.

All these methods (local higher order ABCs, nonlocal ABCs, and PMLs) have been successfully introduced in a number of different computational codes. Of course, for anybody who wants to use such codes, the natural question is, Which is the best method for the absorption of waves? Our feeling is that there is no universal answer to such a question and that a response should include some criteria: nature of the problem to be addressed, accuracy, speed of calculation, ease of implementation, long time behavior, etc. However, even with given criteria, the answer would be delicate, in particular because no complete and fair comparison has been done between the three classes of methods. The first reason, which is easy to understand, is that there is probably nobody in the world who has implemented the three methods with the same amount of care. The second reason is a lack of analysis, which is hard in particular if one is interested in getting convincing error estimates. The objective of the present paper is to fill partially this lack in the theory in the case of local ABCs.

Of course, there are a lot of available theoretical results about higher order ABCs. The first question that was raised by Engquist and Majda in their original papers was that of the well-posedness of the IBVP "wave equation-ABC." This is not a trivial question since it is known that polynomial approximations of degree greater than 2 of the function $f(z)$ (as, for instance, the successive Taylor approximations of $f$ around 0 ) give rise to strongly ill-posed problems. However, thanks to the well-known Kreiss theory (the so-called normal mode analysis [26], [22]), the stability theory of higher order $\mathrm{ABCs}$ is more or less completely understood. In particular, necessary and sufficient conditions were given in [27] about the rational approximations of $f(z)$ in order to ensure the strong well-posedness of the corresponding IBVP (of course, the approximations $f_{n}$ given by (1.9) fulfill these conditions, as already observed in [12]) and energy estimates (giving rise to stability results, i.e., a priori estimates independent of $N$ ) were obtained in [8].

Concerning the accuracy of ABCs, the simplest analysis consists in analyzing the reflection of plane waves, which amounts to studying particular solutions of the following form $\left(k \in \mathbb{R}\right.$ and $\theta \in\left[-\frac{\pi}{2}, \frac{\pi}{2}\right]$ are parameters, $K$ is the wave number, while $\theta$ represents the angle of incidence of the incident plane wave):

$$
u_{\theta}(x, t)=\exp i k\left(x_{1} \sin \theta-x_{2} \cos \theta-c t\right)+\mathbf{R} \exp i k\left(x_{1} \sin \theta+x_{2} \cos \theta-c t\right),
$$

where 


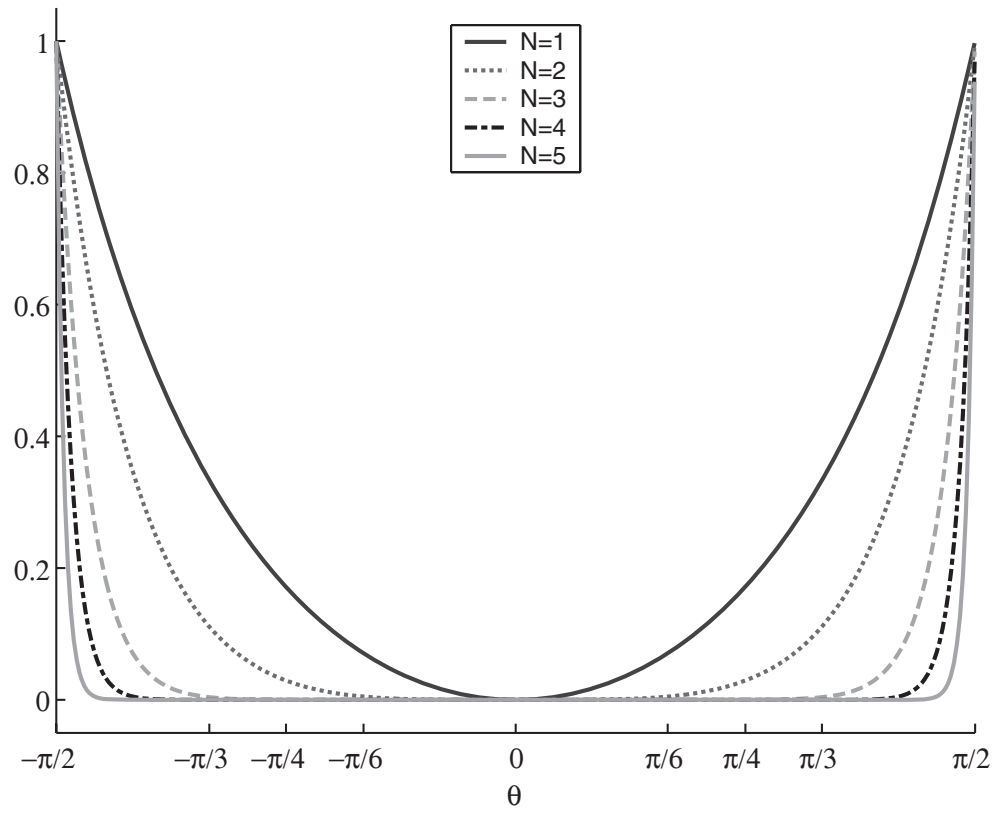

FIG. 1. The reflection coefficient.

(i) $\exp i k\left(x_{1} \sin \theta-x_{2} \cos \theta-c t\right)$ is the incident wave,

(ii) $\exp i k\left(x_{1} \sin \theta+x_{2} \cos \theta-c t\right)$ is the reflected wave, $\mathbf{R}$ being the reflection coefficient.

By construction, (1.11) is a solution of the wave equation (1.1). It remains to determine $\mathbf{R}$ in order to satisfy the boundary condition (1.2). The computations show that $\mathbf{R}$ depends only on the angle of incidence $\theta$ :

$$
\mathbf{R}=\mathbf{R}_{N}(\theta) \equiv \frac{\left(f_{N}-f\right)(\sin \theta)}{\left(f_{N}+f\right)(\sin \theta)}=(-1)^{N}\left(\frac{1-\cos \theta}{1+\cos \theta}\right)^{N}
$$

In particular one sees that for any $\theta \in]-\frac{\pi}{2}, \frac{\pi}{2}\left[, \mathbf{R}_{N}(\theta)\right.$ tends (exponentially fast) to 0 when $N \rightarrow+\infty$ while $\left|\mathbf{R}_{N}\left( \pm \frac{\pi}{2}\right)\right|=1$ (see also Figure 1). There are much fewer results about convergence and error estimates. In fact, there was no real progress since the initial result of Engquist and Majda, which we are recalling now. They were addressing the following $2 \mathrm{D}$ model problem:

$$
\begin{cases}\text { Find } v: \mathbb{R}_{-}^{2} \times \mathbb{R} \mapsto \mathbb{R} & \text { such that } \\ \frac{1}{c^{2}} \frac{\partial^{2} v}{\partial t^{2}}-\Delta v=0 & \text { in } \mathbb{R}_{-}^{2} \times \mathbb{R}^{+}, \\ v\left(x_{1}, 0, t\right)=g\left(x_{1}, t\right) & \text { on } x_{2}=0, \\ v(x, t)=0 & \text { for } t<0 .\end{cases}
$$

One wishes to get a good approximation of $v$ in a domain $\Omega_{b}=\left\{x /-b<x_{2}<0\right\}$ for 
given $b>0$ by putting an $\mathrm{ABC}$ on the line $x_{2}=-a$, with $a>b$ :

$$
\begin{cases}\text { Find } v^{N}: \Omega_{a} \times \mathbb{R} \mapsto \mathbb{R} & \text { such that } \\ \frac{1}{c^{2}} \frac{\partial^{2} v^{N}}{\partial t^{2}}-\Delta v^{N}=0 & \text { in } \Omega_{a} \times \mathbb{R}^{+}, \\ v^{N}\left(x_{1}, 0, t\right)=g\left(x_{1}, t\right) & \text { on } x_{2}=0, \\ \mathcal{B}^{N} v^{N}=0 & \text { on } x_{2}=-a, \\ v^{N}(x, t)=0 & \text { for } t<0 .\end{cases}
$$

In (1.14) there are two important parameters: the order $N$ of the boundary condition and the distance $a$ from the source $g$ to the interface. One assumes that the function $g$ is square integrable in both space and time:

$$
\int_{0}^{+\infty} \int_{\mathbb{R}}\left|g\left(x_{1}, t\right)\right|^{2} d x_{1} d t<+\infty .
$$

TheOREM 1.3 (see [12]). For any $\varepsilon>0$ and any arbitrarily large integer $M$, there exist $N_{0}=N_{0}(\varepsilon, M)$ and $a_{0}=a_{0}(\varepsilon, M)$ such that, for any $N \geq N_{0}$ and $a \geq a_{0}$,

$$
\int_{0}^{T} \int_{\Omega_{b}}\left|\left(v-v^{N}\right)(x, t)\right|^{2} d x d t<\varepsilon \quad \forall T \leq M a .
$$

(i) This result is only a convergence result and does not provide an error estimate. Thus it is not a guide for choosing in practice $N$ and $a$.

(ii) The fact that the result is valid for any time interval of the form $[0, M a]$ indicates that the result takes into account an arbitrary large number of reflections on the absorbing boundary.

(iii) What is not satisfactory with Theorem 1.3 is the fact that the estimate (1.16) requires $a$ to be sufficiently large. In particular, this does not provide a convergence result when $N \rightarrow+\infty$ for fixed $a$.

(iv) Looking at the proof of the theorem enlightens the need for $a$ sufficiently large. It is not our purpose to reproduce here the proof, but it seems useful to emphasize some points. The idea is to use the Fourier transform in space and time:

$$
v\left(x_{1}, x_{2}, t\right) \rightarrow \widetilde{\mathbf{v}}\left(k, x_{2}, \omega\right)=\widetilde{v}\left(k, x_{2}, i \omega\right) .
$$

One can get an explicit solution for both $\widetilde{\mathbf{v}}$ and $\widetilde{\mathbf{v}}^{N}$. In particular, we have

$$
\mid \begin{aligned}
& \widetilde{\mathbf{v}}\left(k, x_{2}, \omega\right)=\widetilde{\mathbf{g}}(k, \omega) \exp \left(k^{2}-\omega^{2} / c^{2}\right)^{\frac{1}{2}} \cdot x_{2}, \\
& \left(k^{2}-\omega^{2} / c^{2}\right)^{\frac{1}{2}}= \begin{cases}\sqrt{k^{2}-\omega^{2} / c^{2}} & \text { if } k^{2} \geq \omega^{2} / c^{2}, \\
i \sqrt{\omega^{2} / c^{2}-k^{2}} & \text { if } k^{2} \leq \omega^{2} / c^{2} .\end{cases}
\end{aligned}
$$

In particular,

(i) if $k^{2}<\omega^{2} / c^{2}$, the function $x_{2} \rightarrow \widetilde{\mathbf{v}}\left(k, x_{2}, \omega\right)$ is oscillating: this is the region of propagative modes;

(ii) if $k^{2}>\omega^{2} / c^{2}$, the function $x_{2} \rightarrow \widetilde{\mathbf{v}}\left(k, x_{2}, \omega\right)$ is exponentially decaying when $x_{2} \rightarrow-\infty$ : this is the region of evanescent modes. 
When one looks at the error $e^{N}=v^{N}-v$, its Fourier transform appears as a sum (the sum is a priori infinite but becomes finite if one is interested in times less than $M a)$ over $j \geq 1$ of terms of the form

$$
\mathcal{R}_{N}\left(\frac{c k}{\omega}\right)^{j} \cdot \widetilde{\mathbf{g}}(k, \omega) \cdot \exp \left[\left(k^{2}-\omega^{2} / c^{2}\right)^{\frac{1}{2}}\left( \pm x_{2}+2 j a\right)\right]
$$

where the reflection coefficient $\mathcal{R}_{N}$ is given by

$$
\mathcal{R}_{N}(\nu)=\frac{\left(f_{N}-f\right)(\nu)}{\left(f_{N}+f\right)(\nu)}
$$

and satisfies

$$
\begin{cases}\mathcal{R}_{N}(\sigma) \leq 1 & \text { (stability result) } \\ \mathcal{R}_{N}(\sigma) \rightarrow 0 & \text { for }|\sigma|<1 \text { (cf. Remark 1.2) }\end{cases}
$$

Up to technical details (this is in particular where the assumption (1.15) intervenes), the idea of the proof is the following:

(i) In the propagative region $k^{2}<\omega^{2} / c^{2},\left|\mathcal{R}_{N}(\sigma)\right|^{j}$ can be made arbitrarily small by choosing $N$ large enough.

(ii) In the evanescent region $k^{2}>\omega^{2} / c^{2},\left|\exp \left[\left(k^{2}-\omega^{2} / c^{2}\right)^{\frac{1}{2}}\left( \pm x_{2}+2 j a\right)\right]\right|$ can be made arbitrarily small by choosing $a$ large enough.

One then concludes with Plancherel's theorem.

Physically, the fact that $f_{n}(z)$ has nothing to do with $f(z)$ for $|z|>1$ means that the evanescent modes are not correctly taken into account by the absorbing condition. This is why one needs to have $a$ large enough in order to "kill" the amplitude of the evanescent modes at the boundary $x_{2}=a$.

In 1988, Halpern and Rauch proposed a high-frequency analysis in [20]. More recently, an advance was achieved by Hagström (see [17] and [16], [18]), who derived an approximation theory for the approximation of (a class of) pseudodifferential operators, with the aim of applying it to ABCs, based on a new reinterpretation of (1.2) and standard quadrature theory. He obtained error estimates and convergence results only by making $N$ go to $+\infty$ (i.e., without touching the position of the boundary). However, its results were nonuniform in time.

The history of the present work is the following. The Cagniard-De Hoop method is particularly well known in the physics and engineering communities for calculating analytical solutions of time-dependent wave propagation problems, especially in seismology (see [7], [25], [24]). This method permits one, moreover, to establish a link between time domain solutions and harmonic plane waves. Trying to learn something about this method (for a completely different problem), we immediately realized that it could easily be applied to the problem of ABCs and would probably help to get new error estimates. The computations are so simple that it is rather surprising to see that nobody did them before, to our knowledge. This article presents the results we have obtained with this method and may prove to be a useful tool in teaching this subject.

The outline of the paper is as follows. In section 2, we describe the model problem we are dealing with (the half-space problem with a point source) and state our two main results: Theorem 2.1, which provides an explicit solution of the corresponding fundamental solution, and Theorem 2.4, which provides error estimates in the case 
of a general source function. These two results show that one can get a convergence result only by letting $N$ go to $+\infty$. In some sense, this shows that the need for large $a$ in Theorem 1.3 is due to the technique used in the proof but does not correspond to a necessity. However, our results in Theorems 2.1 and 2.4 show that increasing the distance from the source to the absorbing boundary helps to get better error estimates. We also pay attention to large time behavior of the error, which has already been the subject of previous research works (see [10], [9], [4]). Sections 3 and 4 are devoted to the proofs of Theorems 2.1 and 2.4. In section 5, we analyze our results in more detail and make the comparison between numerical results and (quasi-)analytical results.

2. Main results. The first result of this paper is an explicit expression of the fundamental solution of the $2 \mathrm{D}$ wave equation in the half-space $\mathbb{R}_{2}^{+}=\left\{x_{2}>0\right\}$ with higher order $\mathrm{ABCs}$ on $\Gamma=\left\{x_{2}=0\right\}\left(=\partial \mathbb{R}_{2}^{+}\right)$. Since the problem is invariant under translations in the $x_{1}$-direction, we can restrict ourselves to the case where the source point is

$$
x_{S}=(0, h) \quad \text { with } h>0 .
$$

The problem we want to solve is

$$
\begin{cases}\text { Find } u: \mathbb{R}_{+}^{2} \times \mathbb{R} \mapsto \mathbb{R} & \text { such that } \\ \frac{1}{c^{2}} \frac{\partial^{2} u}{\partial t^{2}}-\Delta u=\delta\left(x-x_{S}\right) \times \delta(t) & \text { in } \mathbb{R}_{+}^{2}, \\ \mathcal{B}^{N} u=0 & \text { on } \Gamma, \\ u(x, t)=0 & \text { for } t<0 .\end{cases}
$$

To state our result, it is useful to introduce some notation. Let us define the image source point $x_{S}^{*}$ by

$$
x_{S}^{*}=(0,-h)
$$

and let us set (see Figure 2)

$$
r(x)=\left|x-x_{S}\right|, \quad r^{*}(x)=\left|x-x_{S}^{*}\right| .
$$

We also define the function $\theta(x), x \in \mathbb{R}_{+}^{2}$, by

$$
\theta(x) \in]-\frac{\pi}{2}, \frac{\pi}{2}\left[, \quad x-x_{S}^{*}=\left(r^{*}(x) \sin \theta(x), r^{*}(x) \cos \theta(x)\right)^{t},\right.
$$

and finally the function $\Phi(x, t), x \in \mathbb{R}_{+}^{2}, t>0$, by

$$
\Phi(x, t)=\frac{r^{*}(x)^{2} \sin ^{2} \theta(x)-\left(c^{2} t^{2}-r^{*}(x)^{2}\right)}{r^{*}(x)^{2} \sin ^{2} \theta(x)+\left(c^{2} t^{2}-r^{*}(x)^{2}\right)}=\frac{x_{1}^{2}-\left(c^{2} t^{2}-r^{*}(x)^{2}\right)}{x_{1}^{2}+\left(c^{2} t^{2}-r^{*}(x)^{2}\right)} .
$$

We can notice that

$$
c t>r^{*}(x) \Longrightarrow|\Phi(x, t)|<1 .
$$

Finally, we recall that the Chebyshev polynomials $P_{N}(x), N \geq 0$, are defined by

$$
P_{0}(x)=1, \quad P_{1}(x)=x, \quad P_{N+1}(x)-2 x P_{N}(x)+P_{N-1}(x)=0
$$




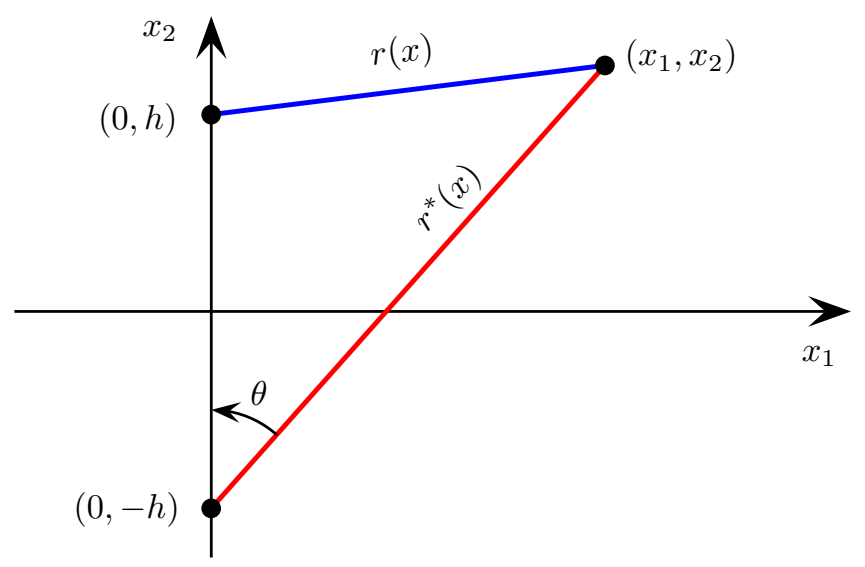

FiG. 2. Illustration of the notation

and satisfy

$$
\forall x \in[-1,1], \quad P_{N}(x)=\cos (N \arccos (x)) .
$$

In particular, we see that

$$
\forall x \in[-1,1], \quad\left|P_{N}(x)\right| \leq 1 .
$$

Theorem 2.1. The solution $u(x, t)=G^{N}(x, t)$ of problem (2.2) is given by

$$
G^{N}(x, t)=G_{i}(x, t)+G_{r}^{N}(x, t),
$$

where, if $H$ denotes the Heaviside function,

$$
\mid \begin{aligned}
& G_{i}(x, t)=\frac{1}{2 \pi \sqrt{t^{2}-\frac{r(x)^{2}}{c^{2}}}} H(c t-r(x)), \\
& G_{r}^{N}(x, t)=-\frac{P_{N}(\Phi(x, t))}{2 \pi \sqrt{t^{2}-\frac{r^{*}(x)^{2}}{c^{2}}}}\left[\frac{c t-\left(x_{2}+h\right)}{c t+\left(x_{2}+h\right)}\right]^{N} H\left(c t-r^{*}(x)\right) .
\end{aligned}
$$

Remark 2.2. The function $G_{i}(x, t)$, which does not depend on $N$, is nothing but the restriction to the half-space $\mathbb{R}_{+}^{2}$ of the fundamental solution of the $2 \mathrm{D}$ wave equation in the whole space. That is why it is called the incident field. Conversely, the field $G_{r}^{N}(x, t)$, due to the presence of the boundary $\Gamma$, is called the reflected field, which does depend on $N$.

Remark 2.3. The presence of the factor $H\left(c t-r^{*}(x)\right)$ indicates that the reflected field $G_{r}^{N}(\cdot, t)$ is compactly supported in the set $\Omega(t)=\Omega_{1}(t) \cup \Omega_{2}(t)$ (see Definition 4.6).

Let us now consider the approximation in the upper half-space of the solution $u$ of the $2 \mathrm{D}$ wave equation with a "smooth" point source:

$$
\begin{cases}\text { Find } u: \mathbb{R}^{2} \times \mathbb{R}^{+} \mapsto \mathbb{R} & \text { such that } \\ \frac{1}{c^{2}} \frac{\partial^{2} u}{\partial t^{2}}-\Delta u=\delta\left(x-x_{S}\right) \times f(t) & \text { in } \mathbb{R}^{2} \times \mathbb{R}^{+}, \\ u(x, 0)=0, \quad \frac{\partial u}{\partial t}(x, 0)=0 & \text { in } \mathbb{R}^{2}\end{cases}
$$


where we assume that the source function $f(t)$ is bounded and has support $[0, T](T$ can be equal to $+\infty$, which includes the case of a permanent source term) by the solution $u^{N}$ of the boundary value problem

$$
\begin{cases}\text { Find } u^{N}: \mathbb{R}^{2} \times \mathbb{R}^{+} \mapsto \mathbb{R} & \text { such that } \\ \frac{1}{c^{2}} \frac{\partial^{2} u^{N}}{\partial t^{2}}-\Delta u^{N}=\delta\left(x-x_{S}\right) \times f(t) & \text { in } \mathbb{R}^{2} \times \mathbb{R}^{+}, \\ \mathcal{B}^{N} u^{N}=0 & \text { on } \Gamma, \\ u(x, 0)=0, \quad \frac{\partial u}{\partial t}(x, 0)=0 & \text { in } \mathbb{R}^{2} .\end{cases}
$$

TheOREM 2.4. At each point $x \in \mathbb{R}_{+}^{2}$, one has the following pointwise estimates:

(i) For $\frac{r^{*}(x)}{c} \leq t \leq \frac{r^{*}(x)}{c}+T\left(\Leftrightarrow x \in \Omega_{1}(t)\right.$ - see (4.6)),

$$
\begin{aligned}
& \left|u(x, t)-u^{N}(x, t)\right| \\
& \quad \leq \frac{1}{2 \pi}\left(\frac{c t-\left(x_{2}+h\right)}{c t+\left(x_{2}+h\right)}\right)^{N} \log \left(\frac{c t+\sqrt{c^{2} t^{2}-r^{*}(x)^{2}}}{r^{*}(x)}\right)\|f\|_{L^{\infty}} .
\end{aligned}
$$

(ii) For $t>\frac{r^{*}(x)}{c}+T\left(\Leftrightarrow x \in \Omega_{2}(t)-\right.$ see $\left.(4.6)\right)$,

$$
\begin{aligned}
& \left|u(x, t)-u^{N}(x, t)\right| \\
& \quad \leq \frac{1}{2 \pi}\left(\frac{c t-\left(x_{2}+h\right)}{c t+\left(x_{2}+h\right)}\right)^{N} \log \left(\frac{c t+\sqrt{c^{2} t^{2}-r^{*}(x)^{2}}}{c(t-T)+\sqrt{c^{2}(t-T)^{2}-r^{*}(x)^{2}}}\right)\|f\|_{L^{\infty}} .
\end{aligned}
$$

Moreover, one has the following uniform estimates:

(i) For $\frac{h}{c} \leq t \leq \frac{h}{c}+T$,

$$
\left\|\left(u-u^{N}\right)(\cdot, t)\right\|_{L^{\infty}\left(\mathbb{R}_{+}^{2}\right)} \leq \frac{1}{2 \pi}\left(\frac{c t-h}{c t+h}\right)^{N} \log \left(\frac{t+\sqrt{t^{2}-(h / c)^{2}}}{(h / c)}\right)\|f\|_{L^{\infty}} .
$$

(ii) For $t>\frac{h}{c}+T$,

$$
\left\|\left(u-u^{N}\right)(\cdot, t)\right\|_{L^{\infty}\left(\mathbb{R}_{+}^{2}\right)} \leq \frac{1}{2 \pi}\left(\frac{c t-h}{c t+h}\right)^{N} \log \left(\frac{t+\sqrt{t^{2}-(t-T)^{2}}}{t-T}\right)\|f\|_{L^{\infty}} .
$$

These results lead to the following comments:

(i) The error converges spectrally to 0 (in the uniform norm) when $N$ goes to infinity.

(ii) For given $t$, the upper bounds in the estimates (2.15) and (2.16) diminish when the distance $h$ from the source to the absorbing boundary increases. This is coherent with the physical intuition and numerical observations.

(iii) Concerning the behavior of the error for large $t$, if we assume that $T<+\infty$, we observe that the right-hand side in the estimate (2.16) behaves for large $t$ as

$$
\frac{1}{2 \pi} \sqrt{\frac{2 T}{t}}
$$


which shows that, for all $N$ and $h$, the error converges uniformly to 0 when $t$ tends to $+\infty$. On the other hand, when $T=+\infty$, the right-hand side in the estimate (2.15) behaves as

$$
\frac{1}{2 \pi} \log t,
$$

which a priori authorizes a logarithmic growth on the error when $t$ tends to $+\infty$. This is what happens if $f(t)$ is, for instance, the Heaviside function.

Remark 2.5. We have chosen here to analyze the approximation of a problem associated to a point source. It would not be difficult to adapt Theorem 2.4 (or more precisely its proof) to treat the case of a distributed source term $f(x, t)$ or nonzero initial data $u_{0}$ and $u_{1}$. In the same way, we have chosen to present $L^{\infty}$ estimates, which seemed to us more pertinent in practice. However, once again, it is easy to adapt the proof in order to get $L^{p}$ or energy estimates.

3. Proof of Theorem 2.1. As we have already stated, the formula (2.9), (2.10) results directly from the application of the Cagniard-De Hoop method to problem (2.2). In order to make this paper easily understandable to a reader who is not familiar with this technique, we detail the proof (only some explicit calculations will be omitted). Let us decompose the solution $u$ of (2.2) as

$$
u=G_{i}+u^{r},
$$

where $G_{i}$, given by $(2.10)$, is the fundamental solution of the $2 \mathrm{D}$ wave equation. By linearity, it is clear that $u^{r}$ satisfies

$$
\begin{cases}\text { Find } u^{r}: \mathbb{R}_{+}^{2} \times \mathbb{R} \mapsto \mathbb{R} & \text { such that } \\ \frac{1}{c^{2}} \frac{\partial^{2} u^{r}}{\partial t^{2}}-\Delta u^{r}=0 & \text { in } \mathbb{R}_{2}^{+}, \\ \mathcal{B}^{N} u^{r}=-\mathcal{B}^{N} G_{i} & \text { on } \Gamma, \\ u(x, t)=0 & \text { for } t<0 .\end{cases}
$$

We apply the following successively to $u^{r}$ :

(i) The Laplace transform in time ( $s$ is the dual variable of $t$ ):

$$
\widetilde{u}^{r}\left(x_{1}, x_{2}, s\right)=\int_{0}^{+\infty} u^{r}\left(x_{1}, x_{2}, t\right) e^{-s t} d t .
$$

(ii) The Fourier transform in the tangential space variable $x_{1}$ ( $k$ is the dual variable of $\left.x_{1}\right)$ :

$$
\widehat{u}^{r}\left(k, x_{2}, s\right)=\int_{-\infty}^{+\infty} \widetilde{u}^{r}\left(x_{1}, x_{2}, s\right) e^{i k x_{1}} d x_{1} .
$$

The algorithm for applying the Cagniard-De Hoop method is the following:

1. Compute explicitly $\widehat{u}^{r}\left(k, x_{2}, s\right)$.

2. Apply the inverse Fourier transform in $x_{1}$ :

$$
\widetilde{u}^{r}\left(x_{1}, x_{2}, s\right)=\frac{1}{2 \pi} \int_{-\infty}^{+\infty} \widehat{u}^{r}\left(k, x_{2}, s\right) e^{-i k x_{1}} d x_{1} .
$$


3. Transform (by means of complex analysis methods) the integral (3.4) into an integral of the form

$$
\widetilde{u}^{r}\left(x_{1}, x_{2}, s\right)=\int_{0}^{+\infty} F\left(x_{1}, x_{2}, t\right) e^{-s t} d t
$$

Then, by surjectivity of the Laplace transform, we shall have identified the solution (compare (3.2) and (3.5))

$$
u^{r}\left(x_{1}, x_{2}, t\right) \equiv F\left(x_{1}, x_{2}, t\right) .
$$

The first step is straightforward. From the wave equation, we deduce that the function $x_{2} \mapsto \widehat{u}^{r}\left(k, x_{2}, s\right)$ satisfies

$$
-\frac{d^{2} \widehat{u}^{r}}{d x_{2}^{2}}+\left(k^{2}+\frac{s^{2}}{c^{2}}\right) \widehat{u}^{r}=0 .
$$

Retaining only the solutions that decay when $x_{2} \rightarrow+\infty$ for $\mathcal{R} e(s) \geq 0$, we deduce the existence of a complex-valued function $A(k, s)$ such that

$$
\widehat{u}^{r}\left(k, x_{2}, s\right)=A(k, s) e^{-\left(k^{2}+\frac{s^{2}}{c^{2}}\right)^{\frac{1}{2}} x_{2}},
$$

where we have chosen to use the determination of the complex square root corresponding to

$$
\forall z \in \mathbb{C}, \quad \mathcal{R} \text { e } z^{\frac{1}{2}} \geq 0
$$

which corresponds to making the branch cut of $z^{\frac{1}{2}}$ coincide with the semireal axis $\operatorname{Im} z=0, \mathcal{R} e z<0$ (see Figure 3). Since $u^{r}+G_{i}$ is smooth for $y<h$, we can use the fact that

$$
\mathcal{B}^{N}\left(u^{r}+G_{i}\right)=0 \Longleftrightarrow\left(\frac{1}{c} \frac{\partial}{\partial t}-\frac{\partial}{\partial x_{2}}\right)^{N}\left(u^{r}+G_{i}\right)=0 \quad \text { for } x_{2}=0 .
$$

On the other hand, it is well known that the Laplace-Fourier transform of $G_{i}$ is given by

$$
\widehat{G}_{i}\left(k, x_{2}, s\right)=\frac{e^{-\left(k^{2}+\frac{s^{2}}{c^{2}}\right)^{\frac{1}{2}}\left|x_{2}-h\right|}}{2\left(k^{2}+\frac{s^{2}}{c^{2}}\right)^{\frac{1}{2}}} .
$$

Taking into account the form (see (3.7) and (3.10)) of $\widehat{u}^{r}$ and $\widehat{G}_{i}$, the boundary condition (3.9) leads to

$$
\left(\frac{s}{c}+\left(k^{2}+\frac{s^{2}}{c^{2}}\right)^{\frac{1}{2}}\right)^{N} A(k, s)+\frac{1}{2}\left(\frac{s}{c}-\left(k^{2}+\frac{s^{2}}{c^{2}}\right)^{\frac{1}{2}}\right)^{N} \frac{e^{-\left(k^{2}+\frac{s^{2}}{c^{2}}\right)^{\frac{1}{2}} h}}{\left(k^{2}+\frac{s^{2}}{c^{2}}\right)^{\frac{1}{2}}}=0 .
$$

This permits us to compute $A(k, s)$ and finally to get

$$
\widehat{u}^{r}\left(k, x_{2}, s\right)=-R^{N}\left(k, \frac{s}{c}\right) \frac{e^{-\left(k^{2}+\frac{s^{2}}{c^{2}}\right)^{\frac{1}{2}}\left(x_{2}+h\right)}}{2\left(k^{2}+\frac{s^{2}}{c^{2}}\right)^{\frac{1}{2}}},
$$




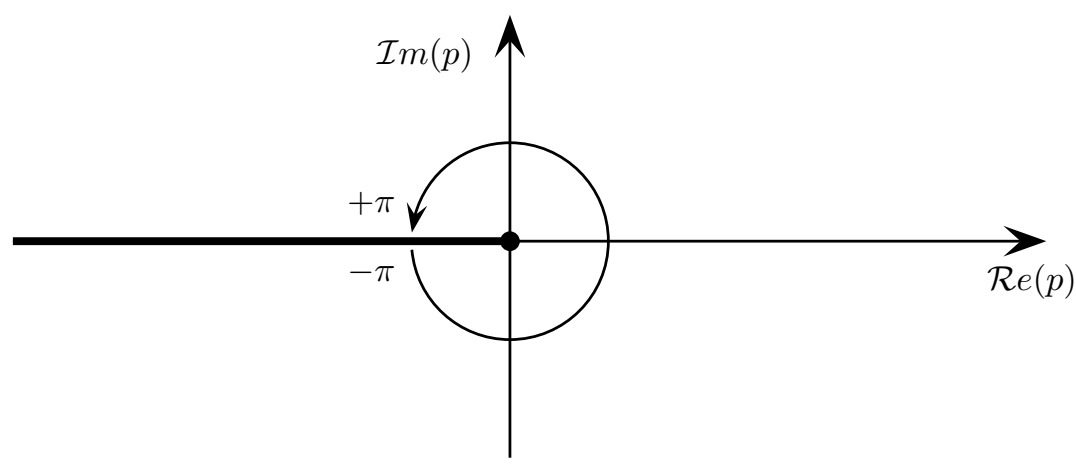

FIG. 3. The branch cut for the determination of the complex square root.

where we have set

$$
R^{N}(k, \sigma)=\mathcal{R}_{N}\left(\frac{k}{\sigma}\right)=\left[\frac{\sigma-\left(k^{2}+\sigma^{2}\right)^{\frac{1}{2}}}{\sigma+\left(k^{2}+\sigma^{2}\right)^{\frac{1}{2}}}\right]^{N} .
$$

Therefore (this is step 2) we have

$$
\widetilde{u}^{r}\left(x_{1}, x_{2}, s\right)=-\frac{1}{4 \pi} \int_{-\infty}^{+\infty} R^{N}\left(k, \frac{s}{c}\right) \frac{e^{-\left(k^{2}+\frac{s^{2}}{c^{2}}\right)^{\frac{1}{2}}\left(x_{2}+h\right)}}{\left(k^{2}+\frac{s^{2}}{c^{2}}\right)^{\frac{1}{2}}} e^{-i k x_{1}} d k
$$

which we would like to transform into an integral of the form (3.5) (this is step 3). This is the part of the approach which is specific to the Cagniard-De Hoop method. We are helped by the following facts:

(i) The integrand in (3.13) is homogeneous in $s$ and $k$.

(ii) The dependence with respect to $x_{2}$ of this integrand is exponential.

First, exploiting the homogeneity property, we apply the change of variable $k=p s / c$ and obtain

$$
\begin{aligned}
& \widetilde{u}^{r}\left(x_{1}, x_{2}, s\right) \\
& \quad=-\frac{1}{4 \pi} \int_{-\infty}^{+\infty} R^{N}(p, 1) \frac{e^{-s\left[\left(1+p^{2}\right)^{\frac{1}{2}}\left(\frac{x_{2}+h}{c}\right)+i p \frac{x_{1}}{c}\right]}}{\left(1+p^{2}\right)^{\frac{1}{2}}} d p, \quad\left(\equiv \int_{-\infty}^{+\infty} \Psi(p) d p\right) .
\end{aligned}
$$

In what follows we fix $\left(x_{1}, x_{2}\right) \in \mathbb{R}_{+}^{2}$ with $x_{1}>0$ (which is not restrictive since the solution we are looking for is clearly even in $x_{1}$ ). We introduce $r^{*}=r^{*}(x)$ and $\theta=\theta(x)\left(\in[0, \pi / 2]\right.$ since $\left.x_{1} \geq 0\right)$ according to the definitions (2.4) and (2.5). We thus have

$$
\left\{\begin{array}{l}
x_{1}=r^{*} \sin \theta \\
x_{2}+h=r^{*} \cos \theta
\end{array}\right.
$$

Now the idea is to consider the variable $p$ as a complex variable and to see the integral (3.14) as a contour integral, the contour coinciding with the real axis. If one is able, by a contour deformation, to transform this integral into a contour integral 


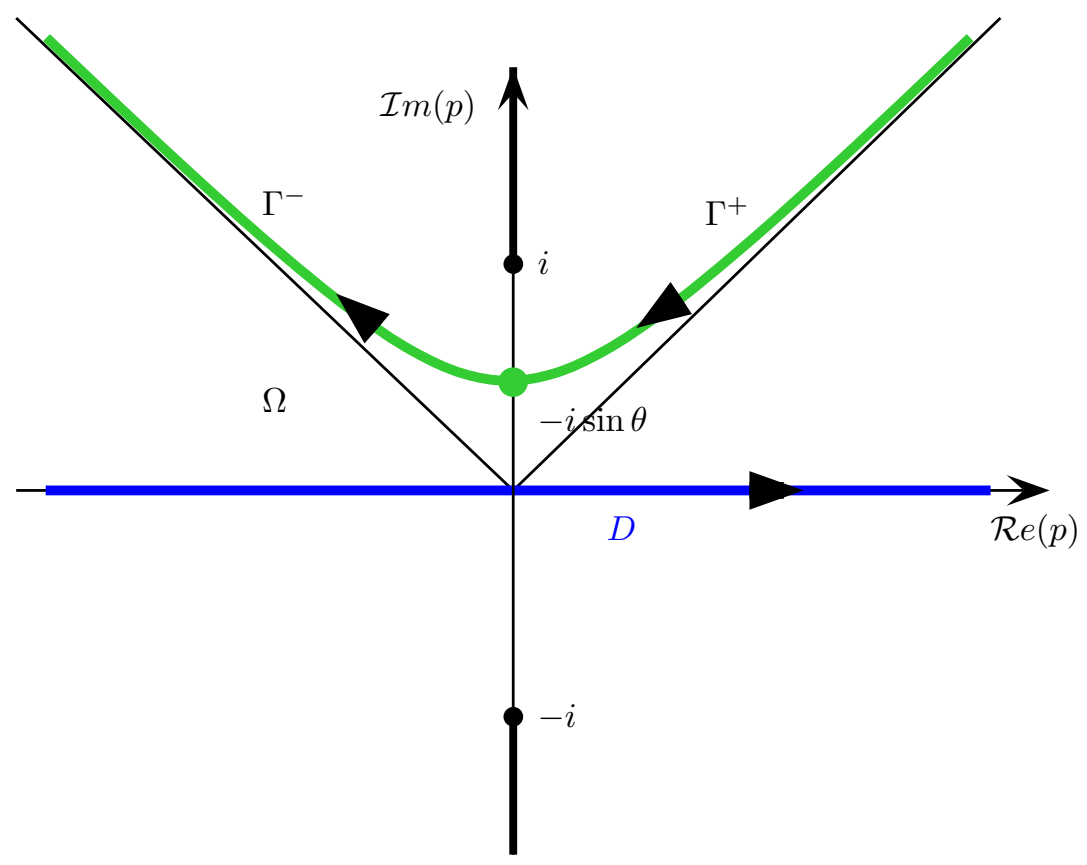

Fig. 4. The contours $\Gamma$ and $D$.

on some curve $\Gamma$ along which one can use a parametric representation of the form

$$
\left(1+p^{2}\right)^{\frac{1}{2}}\left(\frac{x_{2}+h}{c}\right)+i p \frac{x_{1}}{c}=t \quad \text { for } t>0,
$$

we shall have reached our goal. To achieve this, we first remark that the integrand $\Psi(p)$ in (3.14) is an analytic function of $p$ if one excludes the two branch cuts constituted of the two half-lines of purely imaginary numbers whose modulus is greater than 1 (see Figure 4). Then we introduce the so-called Cagniard-De Hoop contour $\Gamma$, defined as

$$
\left\{\begin{array}{l}
\Gamma=\Gamma^{+} \cup \Gamma^{-}, \\
\Gamma^{ \pm}=\left\{p=\gamma^{ \pm}(t) \equiv-i \frac{c t}{r^{*}} \sin \theta \pm \cos \theta \sqrt{\frac{c^{2} t^{2}}{r^{* 2}}-1}, t \geq \frac{r^{*}}{c}\right\} .
\end{array}\right.
$$

It is clear that the two curves $\Gamma^{ \pm}$are symmetric with respect to the imaginary axis, and meet at point $-i \sin \theta$ (for $\left.t=r^{*} / c\right)$. More precisely, it is easy to check that $\Gamma$ is nothing but the branch of the hyperbola of

$$
\frac{Y^{2}}{\sin ^{2} \theta}-\frac{X^{2}}{\cos ^{2} \theta}=1 \quad\left(p=X+i Y,(X, Y) \in \mathbb{R}^{2}\right),
$$

which is located in the upper half-space $Y=\mathcal{I} m p>0$. Note that this hyperbola does not intersect the two branch cuts of $\Psi$. All this information is summarized in Figure 4 .

In fact to understand where (3.17) comes from, it suffices to remark that $\gamma^{ \pm}(t)$ are nothing but the two roots of (3.16), considered as an equation in $p$ (the calculations are left to the reader). 


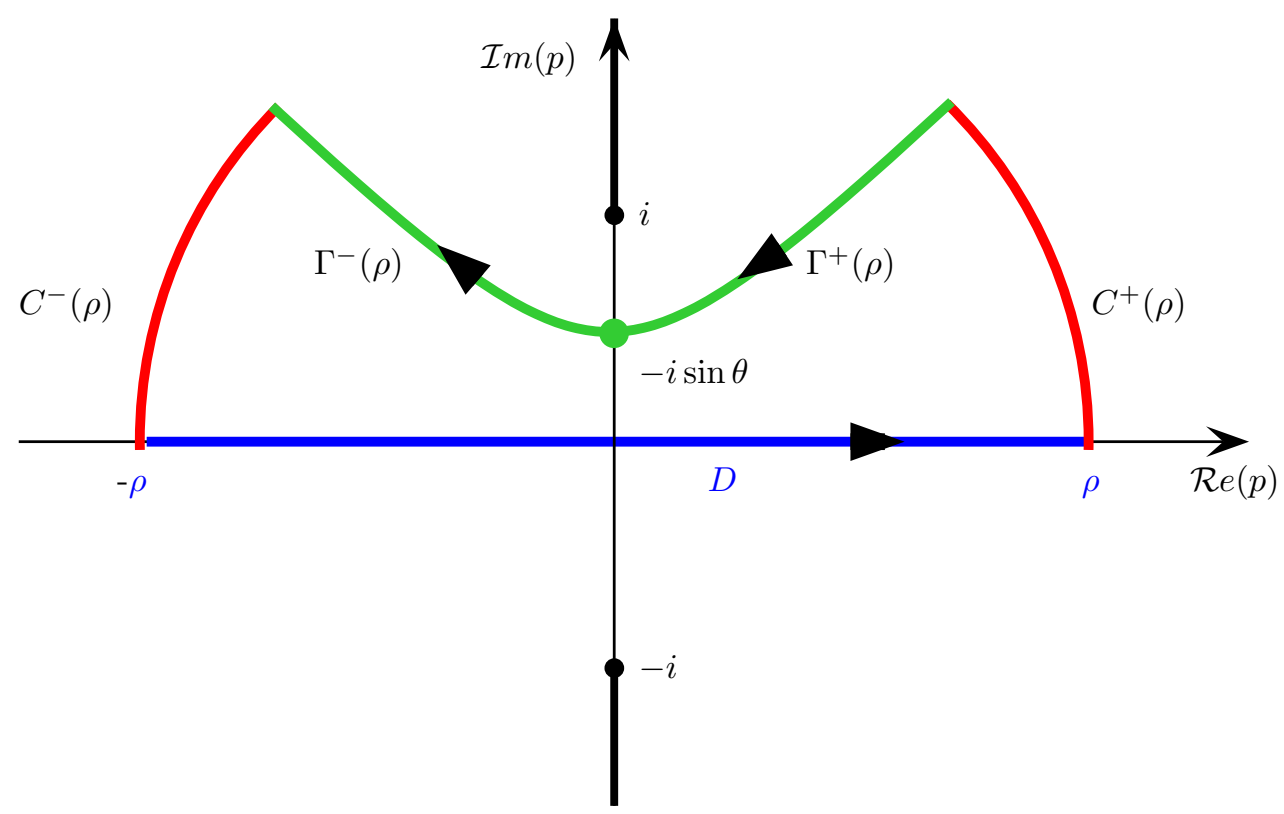

FIG. 5. The closed contour $D_{\rho} \cup C_{\rho} \cup \Gamma_{\rho}$.

Let us denote by $D$ the real line and by $\Omega$ the connected part of the complex plane delimited by $D$ and $\Gamma$. Let $\rho>0$ be a parameter that is meant to tend to $+\infty$. We set

$$
\mid \begin{aligned}
& D_{\rho}=\{p \in D /|p| \leq \rho\}, \quad \Gamma_{\rho}=\{p \in \Gamma /|p| \leq \rho\} \\
& C_{\rho}=\{p \in \Omega /|p|=\rho\} .
\end{aligned}
$$

Note that $C_{\rho}$ is made of two arcs of the circle of center 0 and radius $\rho$ that join $D_{\rho}$ to $\Gamma_{\rho}$ in such a way that $D_{\rho} \cup C_{\rho} \cup \Gamma_{\rho}$ is a closed curve. Since $\Psi(p)$ is analytic in $\Omega$, the integral of $\Psi$ along $D_{\rho} \cup C_{\rho} \cup \Gamma_{\rho}$ (we choose the orientation of this path such that the real segment is described in the sense of increasing values - see Figure 5) is identically 0:

$$
\int_{D_{\rho}} \Psi(p) d p+\int_{C_{\rho}} \Psi(p) d p+\int_{\Gamma_{\rho}} \Psi(p) d p=0 .
$$

Thanks to the choice of the square root, and since $x_{2}+h>0$, the function $\Psi(p)$ decays exponentially to 0 when $\mathcal{I} m p$ goes to $+\infty$. As a consequence, it is easy to show that (Jordan's lemma)

$$
\lim _{\rho \rightarrow+\infty} \int_{C_{\rho}} \Psi(p) d p=0
$$

Therefore, from (3.14), we deduce

$$
\widetilde{u}^{r}\left(x_{1}, x_{2}, s\right)=-\frac{1}{4 \pi} \int_{\Gamma} R^{N}(p, 1) \frac{e^{-s\left[\left(1+p^{2}\right)^{\frac{1}{2}}\left(\frac{x_{2}+h}{c}\right)+i p \frac{x_{1}}{c}\right]}}{\left(1+p^{2}\right)^{\frac{1}{2}}} d p .
$$


We use the parametrizations $p=\gamma^{+}(t)$ and $p=\gamma^{-}(t)$ for $t \geq \frac{r^{*}(x)}{c}$, respectively, along $\Gamma^{+}$and $\Gamma^{-}$and remark that

$$
\begin{aligned}
& \text { - }\left(1+p^{2}\right)^{\frac{1}{2}}\left(\frac{x_{2}+h}{c}\right)+i p \frac{x_{1}}{c}=t \quad \text { (by construction), } \\
& \text { - } \frac{d p}{\left(\frac{1}{c^{2}}+p^{2}\right)^{\frac{1}{2}}}= \pm \frac{d t}{\left(t^{2}-\frac{r^{* 2}}{c^{2}}\right)^{\frac{1}{2}}} \quad \text { on } \Gamma^{ \pm} \text {. }
\end{aligned}
$$

Therefore, since $t$ goes from $+\infty$ to $\frac{r^{*}}{c}$ on $\Gamma^{+}$and from $\frac{r^{*}}{c}$ to $+\infty$ on $\Gamma^{-}$,

$$
\widetilde{u}^{r}\left(x_{1}, x_{2}, s\right)=-\frac{1}{4 \pi} \int_{\frac{r^{*}}{c}}^{+\infty}\left[R^{N}\left(\gamma^{+}(t), 1\right)+R^{N}\left(\gamma^{-}(t), 1\right)\right] \frac{e^{-s t}}{\left(t^{2}-\frac{r^{* 2}}{c^{2}}\right)^{\frac{1}{2}}} d t .
$$

Finally, observing that $\gamma^{-}(t)^{2}={\overline{\gamma^{+}(t)}}^{2}$, and using the fact that $\sqrt{\bar{z}}=\overline{\sqrt{z}}$, we deduce that

$R^{N}\left(\gamma^{-}(t), 1\right)=\overline{R^{N}\left(\gamma^{+}(t), 1\right)} \Longrightarrow R^{N}\left(\gamma^{+}(t), 1\right)+R^{N}\left(\gamma^{-}(t), 1\right)=2 \mathcal{R} e\left[R^{N}\left(\gamma^{+}(t), 1\right)\right]$,

which yields

$$
\widetilde{u}^{r}\left(x_{1}, x_{2}, s\right)=-\frac{1}{2 \pi} \int_{\frac{r^{*}}{c}}^{+\infty} \mathcal{R} e\left[R^{N}\left(\gamma^{+}(t), 1\right)\right] \frac{e^{-s t}}{\left(t^{2}-\frac{r^{*} 2}{c^{2}}\right)^{\frac{1}{2}}} d t .
$$

Thanks to formula (3.12), one has

$$
R^{N}\left(\gamma^{+}(t), 1\right)=\left[R^{1}\left(\gamma^{+}(t), 1\right)\right]^{N}
$$

while one easily computes that

$$
R^{1}\left(\gamma^{+}(t), 1\right)=\frac{r^{*}-c t \cos \theta+i \sin \theta \sqrt{c^{2} t^{2}-r^{* 2}}}{r^{*}+c t \cos \theta-i \sin \theta \sqrt{c^{2} t^{2}-r^{* 2}}}=\rho_{1}(t) e^{i \alpha(t)}
$$

Setting $\Phi=\Phi(x, t)$ (cf. (2.6)), one finds that (the calculations - rather tedious but straightforward - are left to the reader)

$$
\left\{\begin{array}{l}
\rho_{1}(t)=\frac{c t-r^{*} \cos \theta}{c t+r^{*} \cos \theta}=\frac{c t-\left(x_{2}+h\right)}{c t+\left(x_{2}+h\right)}, \\
\cos \alpha(t)=\frac{r^{* 2} \sin ^{2} \theta-\left(c^{2} t^{2}-r^{* 2}\right)}{r^{* 2} \sin ^{2} \theta+\left(c^{2} t^{2}-r^{* 2}\right)}=\Phi .
\end{array}\right.
$$

Therefore, according to (3.19),

$$
\mathcal{R} e\left[R^{N}\left(\gamma^{+}(t), 1\right)\right]=\rho_{1}(t)^{N} \cos (N \alpha(t)),
$$

that is to say, since $\alpha(t)=\arccos \Phi$,

$$
\mathcal{R} e\left[R^{N}\left(\gamma^{+}(t), 1\right)\right]=\left[\frac{c t-\left(x_{2}+h\right)}{c t+\left(x_{2}+h\right)}\right]^{N} P_{N}(\Phi) .
$$

It is then easy to conclude the proof of Theorem 2.1 from (3.18) and (3.20). 
4. Proof of Theorem 2.4. Let $u$ and $u^{N}$ be the respective solutions of (2.11) and (2.12). We introduce the error (or reflected field) $e^{N}$ defined as

$$
e^{N}=u^{N}-u \text {. }
$$

To get the pointwise estimates (2.13) and (2.14), we fix $x \in \mathbb{R}_{+}^{2}$ and set $r^{*}=r^{*}(x)$ and $\theta=\theta(x)$. Obviously $e^{N}(x, t)=0$ for $t \leq r^{*} / c$, while for $t>r^{*} / c$ we deduce from Theorem 2.1 that

$$
e^{N}(x, t)=\int_{\max \left(\frac{r^{*}}{c}, t-T\right)}^{t} G_{r}^{N}(x, \tau) f(t-\tau) d \tau, \quad t \geq \frac{r^{*}}{c},
$$

using the fact that $f$ is supported in $[0, T]$ and $G_{r}^{N}(x, \cdot)$ in $\left[0, \frac{r^{*}}{c}\right]$. We deduce that

$$
\begin{cases}\left|e^{N}(x, t)\right| \leq\|f\|_{L^{\infty}(0, t)} \cdot\left\|G_{r}^{N}(x, \cdot)\right\|_{L^{1}\left(\frac{r^{*}}{c}, t\right)} & \text { if } \frac{r^{*}}{c} \leq t \leq \frac{r^{*}}{c}+T, \\ \left|e^{N}(x, t)\right| \leq\|f\|_{L^{\infty}(0, T)} \cdot\left\|G_{r}^{N}(x, \cdot)\right\|_{L^{1}(t-T, t)} & \text { if } t>\frac{r^{*}}{c}+T .\end{cases}
$$

We thus have to estimate the $L^{1}$-norm of the functions $t \mapsto G_{r}^{N}(\cdot, t)$. Using the fact that

$$
\left|P_{N}(\Phi(x, t))\right| \leq 1
$$

(estimate which is quasi-optimal for a range of values of $t$ ) we get

$$
\left|G_{r}^{N}(x, t)\right| \leq \frac{1}{2 \pi \sqrt{t^{2}-\frac{r^{* 2}}{c^{2}}}}\left(\frac{c t-\left(x_{2}+h\right)}{c t+\left(x_{2}+h\right)}\right)^{N} .
$$

We remark that, for $t \geq \frac{r^{*}}{c}$, the function $c t \mapsto\left(\frac{c t-\left(x_{2}+h\right)}{c t+\left(x_{2}+h\right)}\right)$ is increasing. Therefore,

$$
\begin{aligned}
\left\|G_{r}^{N}(x, \cdot)\right\|_{L^{1}\left(\frac{r^{*}}{c}, t\right)} & \leq \frac{1}{2 \pi}\left(\frac{c t-\left(x_{2}+h\right)}{c t+\left(x_{2}+h\right)}\right)^{N} \int_{\frac{r^{*}}{c}}^{t} \frac{d \tau}{\sqrt{\tau^{2}-\frac{r^{* 2}}{c^{2}}}} \\
& =\frac{1}{2 \pi}\left(\frac{c t-\left(x_{2}+h\right)}{c t+\left(x_{2}+h\right)}\right)^{N} \log \left(\frac{c t+\sqrt{c^{2} t^{2}-r^{* 2}}}{r^{*}}\right),
\end{aligned}
$$

while, as soon as $t>\frac{r^{*}}{c}+T$,

$$
\begin{aligned}
& \left\|G_{r}^{N}(x, \cdot)\right\|_{L^{1}(t-T, t)} \\
& \quad \leq \frac{1}{2 \pi}\left(\frac{c t-\left(x_{2}+h\right)}{c t+\left(x_{2}+h\right)}\right)^{N} \int_{t-T}^{t} \frac{d \tau}{\sqrt{\tau^{2}-\frac{r^{* 2}}{c^{2}}}} \\
& \quad=\frac{1}{2 \pi}\left(\frac{c t-\left(x_{2}+h\right)}{c t+\left(x_{2}+h\right)}\right)^{N} \log \left(\frac{c t+\sqrt{c^{2} t^{2}-r^{* 2}}}{c(t-T)+\sqrt{c^{2}(t-T)^{2}-r^{* 2}}}\right) .
\end{aligned}
$$

It is easy to deduce (2.13) and (2.14) from (4.3), (4.4), and (4.5).

We now move to the proof of the uniform estimates (2.15) and (2.16). Let us introduce the two disjoint sets

$$
\mid \begin{aligned}
& \Omega_{1}(t)=\left\{x \in R_{+}^{2} / c(t-T)<r^{*}(x) \leq c t\right\} \\
& \Omega_{2}(t)=\left\{x \in R_{+}^{2} / r^{*}(x) \leq c(t-T)\right\}
\end{aligned}
$$




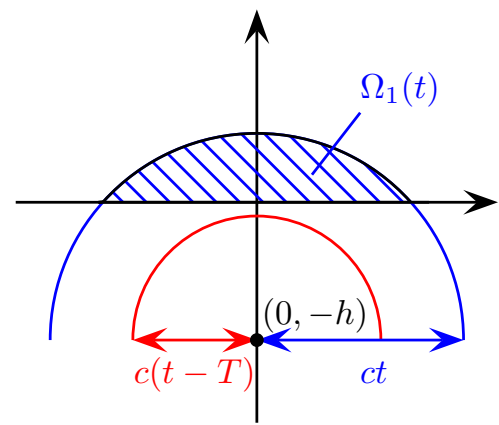

FIG. 6. The set $\Omega_{1}(t), \frac{h}{c} \leq t<\frac{h}{c}+T$.

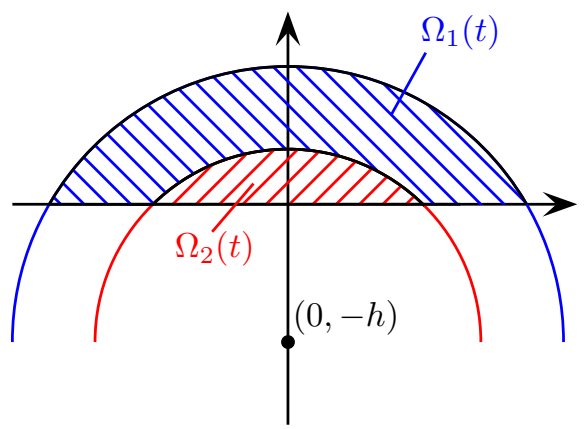

FIG. 7. $\Omega_{1}(t)$ and $\Omega_{2}(t), t \geq \frac{h}{c}+T$.

These two sets are represented in Figures 6 and 7 for two values of $t$. Note that $\Omega_{1}(t)$ is not empty as soon as $t>\frac{h}{c}$, while $\Omega_{2}(t)$ is not empty as soon as $t>\frac{h}{c}+T$. According to (4.3), in order to derive an $L^{\infty}$ estimate of $e^{N}(\cdot, t)$, we need an upper bound for the quantity

$$
\sup _{x \in \Omega_{1}(t)}\left\|G_{r}^{N}(x, \cdot)\right\|_{L^{1}\left(\frac{r^{*}}{c}, t\right)} \quad \text { when } t>\frac{h}{c}
$$

and for the quantity

$$
\sup _{x \in \Omega_{2}(t)}\left\|G_{r}^{N}(x, \cdot)\right\|_{L^{1}(t-T, t)} \quad \text { when } t>\frac{h}{c}+T .
$$

We remark that for each $x \in \Omega(t)=\Omega_{1}(t) \cup \Omega_{2}(t), h \leq x_{2}+h \leq c t$. Therefore, noticing that the function

$$
x \mapsto \frac{c t-x}{c t+x}, \quad x \in[0, c t]
$$

is decreasing, we get

$$
\sup _{x \in \Omega_{1}(t)}\left(\frac{c t-\left(x_{2}+h\right)}{c t+\left(x_{2}+h\right)}\right)=\sup _{x \in \Omega_{2}(t)}\left(\frac{c t-\left(x_{2}+h\right)}{c t+\left(x_{2}+h\right)}\right)=\frac{c t-h}{c t+h} .
$$

On the other hand, using the fact that the two functions

$$
\mid \begin{array}{ll}
r \mapsto \frac{c t}{r}+\sqrt{\frac{c^{2} t^{2}}{r^{2}}-1,} & r \in[0, c t], \\
r \mapsto \frac{c t+\sqrt{c^{2} t^{2}-r^{2}}}{c(t-T)+\sqrt{c^{2}(t-T)^{2}-r^{2}}}, & r \in[0, c(t-T)](t>T),
\end{array}
$$


are, respectively, decreasing and increasing, we deduce that

$$
\begin{aligned}
& \left|\sup _{x \in \Omega_{1}(t)} \log \right| \frac{c t}{r^{*}}+\sqrt{\frac{c^{2} t^{2}}{r^{* 2}}-1} \mid=\log \left(\frac{t+\sqrt{t^{2}-(h / c)^{2}}}{(h / c)}\right) \quad \text { if } \frac{h}{c}<t<\frac{h}{c}+T, \\
& \sup _{x \in \Omega_{1}(t)} \log \left|\frac{c t}{r^{*}}+\sqrt{\frac{c^{2} t^{2}}{r^{* 2}}-1}\right|=\log \left(\frac{t+\sqrt{t^{2}-(t-T)^{2}}}{t-T}\right) \quad \text { if } t>\frac{h}{c}+T, \\
& \sup _{x \in \Omega_{2}(t)} \log \left|\frac{c t+\sqrt{c^{2} t^{2}-r^{* 2}}}{c(t-T)+\sqrt{c^{2}(t-T)^{2}-r^{* 2}}}\right|=\log \left(\frac{t+\sqrt{t^{2}-(t-T)^{2}}}{t-T}\right) \\
& \text { if } t>\frac{h}{c}+T
\end{aligned}
$$

These last three equalities, together with (4.3), (4.4), and (4.5), permit us to show the inequalities

$$
\begin{aligned}
& \sup _{x \in \Omega_{1}(t)}\left\|G_{r}^{N}(x, \cdot)\right\|_{L^{1}\left(\frac{r^{*}}{c}, t\right)} \\
& \quad \leq \frac{1}{2 \pi}\left(\frac{c t-h}{c t+h}\right)^{N} \log \left(\frac{c t}{h}+\sqrt{\frac{c^{2} t^{2}}{h^{2}}-1}\right) \quad\left(t>\frac{h}{c}\right), \\
& \sup _{x \in \Omega_{2}(t)}\left\|G_{r}^{N}(x, \cdot)\right\|_{L^{1}(t-T, t)} \\
& \quad \leq \frac{1}{2 \pi}\left(\frac{c t-h}{c t+h}\right)^{N} \log \left(\frac{t+\sqrt{t^{2}-(t-T)^{2}}}{t-T}\right) \quad\left(t>\frac{h}{c}+T\right) .
\end{aligned}
$$

It is then easy to conclude the proof of Theorem 2.4 from (4.8), (4.9), and (4.3).

Remark 4.1. In formula (2.10), the function $G_{r}^{N}$ naturally appears as the product of three terms. In the proof above, in order to estimate $G_{r}^{N}$ we have estimated independently, for the sake of simplicity, each of these factors. In particular, our final estimates are not necessarily sharp.

\section{Illustration and analysis of the results.}

\subsection{Analysis of the $2 \mathrm{D}$ fundamental solutions.}

Relative error analysis. One of the difficulties in representing numerically the reflected field $G_{r}^{N}$ given by (2.10) is the presence of the singularity of the circle $r^{*}(x)=$ ct. To overcome this difficulty, the idea is to compare this reflected field to what it would be with the Dirichlet boundary condition (which corresponds to $N=0$ ). That is why we introduce the relative error field defined as

$$
\gamma_{r}^{N}(x, t)=\frac{G_{r}^{N}(x, t)}{G_{r}^{0}(x, t)}=P_{N}(\Phi(x, t))\left[\frac{c t-\left(x_{2}+h\right)}{c t+\left(x_{2}+h\right)}\right]^{N}, \quad x \in \Omega(t) .
$$

(Note that $G_{r}^{0}(\cdot, t)$ does not vanish inside the disk $r^{*}(x)<c t$.) In the following experiments we choose $h=1$ and $c=1$. On Figures 8 to 12 we represent, at three different times - namely, $t=3,5$, and 7 from top to bottom - the level lines of $x \mapsto \gamma_{r}^{N}(x, t)$. Each figure corresponds to one value of $N(N=1,2,5,10,20)$.

We clearly observe the following: 




FIG. 8. $x \mapsto \gamma_{r}^{1}(x, t), t=3,5$, and 7 .

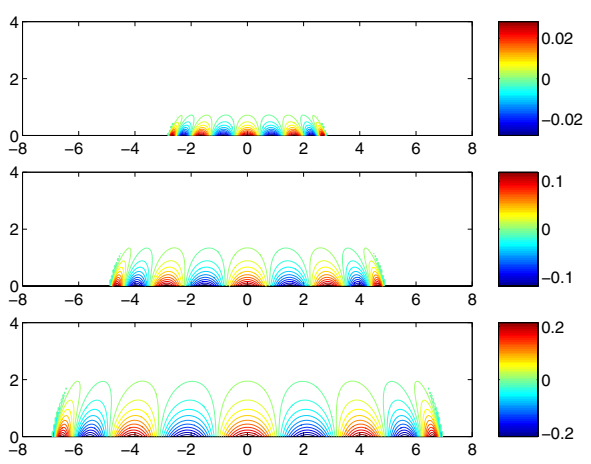

FIG. 10. $x \mapsto \gamma_{r}^{5}(x, t), t=3,5$, and 7 .



FIG. 9. $x \mapsto \gamma_{r}^{2}(x, t), t=3,5$, and 7 .

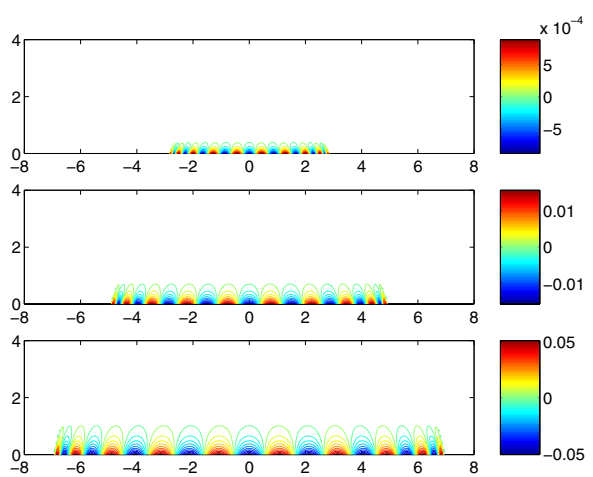

FIG. 11. $x \mapsto \gamma_{r}^{10}(x, t), t=5,7$, and 9 .

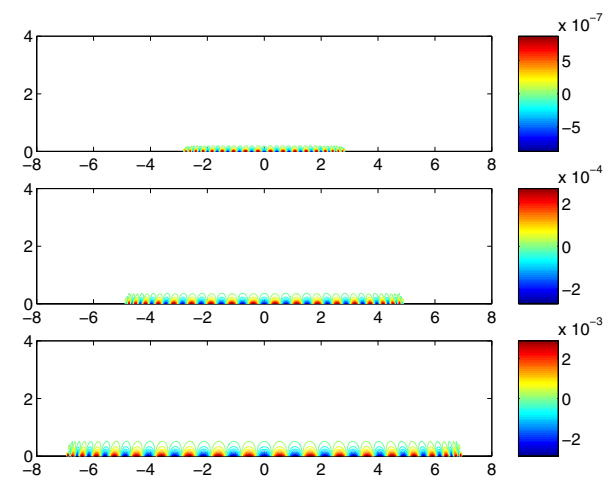

FIG. 12. $x \mapsto \gamma_{r}^{20}(x, t), t=3,5$, and 7 .

(i) The amplitude of the error strongly decays with $N$ (take care of the scales). For instance, at $t=3$, the error level is 0.4 for $N=1,0.2$ for $N=2,0.02$ for $N=5,7.10^{-4}$ for $N=10$, and $6 \cdot 10^{-7}$ for $N=20$.

(ii) As expected, the amplitude of the error also increases in time. By a homogeneity argument, it is obvious that looking at different $t$ 's for fixed $h$ is equivalent to 


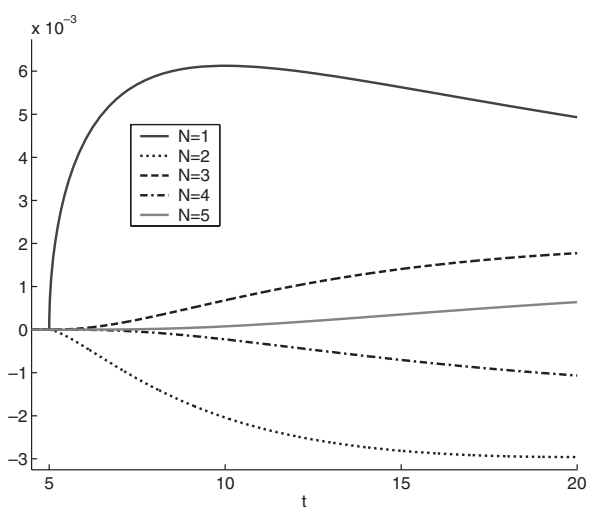

FIG. 13. $t \mapsto G_{r}^{N}(x, t), r^{*}=5, \theta=0$.

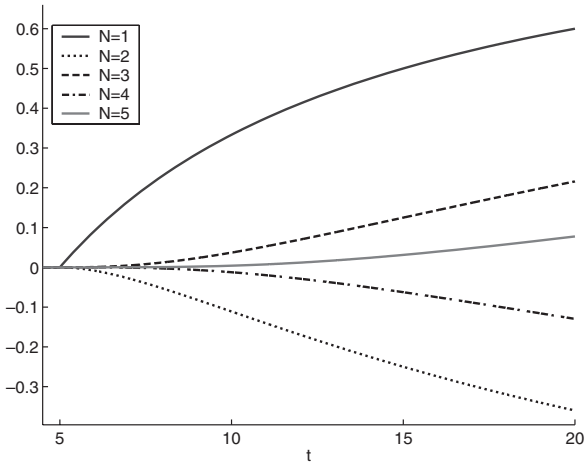

FIG. 14. $t \mapsto \gamma_{r}^{N}(x, t), r^{*}=5, \theta=0$.

looking at different $h$ 's for fixed $t$. Therefore, our results also illustrate the influence of $h$ on the reflected field.

(iii) When $N$ increases, the relative error concentrates more and more on the neighborhood of the absorbing boundary. Moreover, its dependence with respect to the space variable is more and more complicated (this is the effect of the Chebyshev polynomials).

Study of the error as a function of time. Here we wish to study the evolution of the reflected field at a given point $x$ as a function of time. All the points we observe are located on the circle $r^{*}(x)=5$ so that the reflected field arrives at these points at time $t=5$.

(i) The case of the point $\theta(x)=0$. Contrary to what the plane wave analysis might suggest, the reflected field is not identically 0 for $\theta(x)=0$, i.e., on the $x_{2}$ axis. However, the function $t \mapsto G_{r}^{N}(x, t)$ is not discontinuous (except for $N=0$ !) at time $t=\tau=\tau(x)=\left(x_{2}+h\right) / c$, as shown by the formula

$$
G_{r}^{N}(x, t)=\frac{(-1)^{N+1}}{2 \pi} \frac{\left[c t-\left(x_{2}+h\right)\right]^{N-\frac{1}{2}}}{\left[c t+\left(x_{2}+h\right)\right]^{N+\frac{1}{2}}} \quad \text { for } t>\tau .
$$

It becomes even less and less singular as $N$ increases. Moreover, one sees that the function $t \mapsto G_{r}^{N}(x, t)$ is increasing from $t=\tau$ to $t=2 N \tau$, then decreasing for $t>2 N \tau$, and tends to 0 when $t \rightarrow+\infty$ as $1 / 2 \pi c t$. The maximum of $t \mapsto G_{r}^{N}(x, t)$ is given by

$$
\sup _{t \geq \tau} G_{r}^{N}(x, t)=\frac{1}{2 N+1}\left(\frac{2 N-1}{2 N+1}\right)^{N-\frac{1}{2}} \sim \frac{1}{2 N e} \quad(N \rightarrow+\infty) .
$$

These properties are illustrated in Figures 13 and 14, where we represent (in Figure 13) the variations of $t \mapsto G_{r}^{N}(x, t), t \in[0,20]$ for $N=1$ to 5 . Looking at the relative error, the formula

$$
\gamma_{r}^{N}(x, t)=(-1)^{N} \frac{\left[c t-\left(x_{2}+h\right)\right]^{N-\frac{1}{2}}}{\left[c t+\left(x_{2}+h\right)\right]^{N+\frac{1}{2}}} \quad \text { for } t>\tau
$$

shows that the function $t \mapsto \gamma_{r}^{N}(x, t), t>\tau$, increases from 0 to 1 . This is confirmed in Figure 14. 


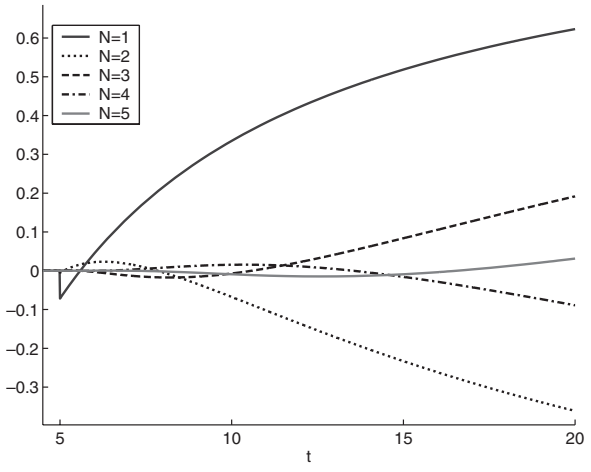

FIG. 15. $t \mapsto \gamma_{r}^{N}(x, t), r^{*}=5, \theta=\frac{\pi}{6}$.

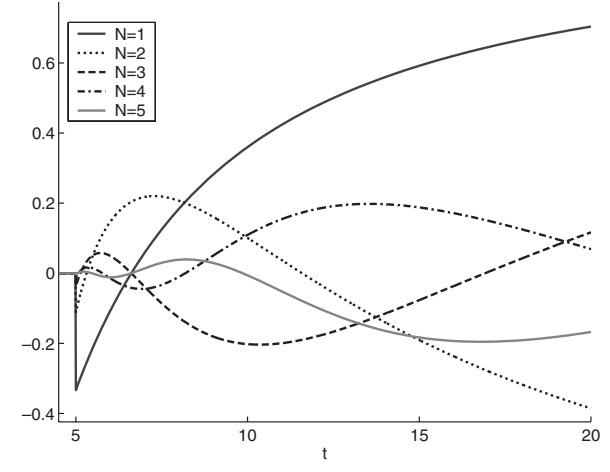

FIG. 16. $t \mapsto \gamma_{r}^{N}(x, t), r^{*}=5, \theta=\frac{\pi}{3}$.

(ii) The case of points $\theta(x) \neq 0$. In this case, the function $t \mapsto \gamma_{r}^{N}(x, t)$ is no longer continuous for $t=\tau$ :

$$
\lim _{t \rightarrow \tau} \gamma_{r}^{N}(x, t)=\mathbf{R}_{N}(\theta(x))=(-1)^{N}\left(\frac{1-\cos \theta}{1+\cos \theta}\right)^{N} .
$$

In Figures 15 and 16 we represent the variations of $t \mapsto \gamma_{r}^{N}(x, t)$ for $\theta=\pi / 6$ and $\theta=\pi / 3$. In each figure, one makes $N$ vary from 1 to 5 . Clearly, the higher $N$ is, the more the function oscillates. Finally, for large times, one easily computes that

$$
\lim _{t \rightarrow+\infty} \gamma_{r}^{N}(x, t)=(-1)^{N}
$$

independently of the value of $N$.

Study of the error as a function of the distance to the image source. We consider here the spatial variation of the reflected field along a ray, namely, the part of a halfline starting from the image source point $S^{*}$ included in the half-space $\mathbb{R}_{+}^{2}$. For a given direction $\theta \in]-\pi / 2, \pi / 2[$, this ray is also defined as

$$
D_{\theta}=\left\{x \in \mathbb{R}_{+}^{2} / \theta(x)=\theta\right\}=\left\{\left(r^{*} \sin \theta, r^{*} \cos \theta\right), r^{*} \geq h / \cos \theta\right\} .
$$

In the following figures we represent the variations of the reflected field $G_{r}^{N}$, for fixed $\theta$ as a function of $r^{*} \geq h / \cos \theta$, for different values of $t$ and $N$.

For $\theta=0, r^{*} \leq h$. In Figures 17 to 19, we represent the variations of $G_{r}^{N}$ along $D_{0}$ for three values of $t, t=3,5,8$. Each figure corresponds to one value of $N$, and therefore the scale varies a lot from one picture to another. Once again, one observes that the reflected field is smoother and smoother as $N$ increases.

For $\theta=\pi / 6, r^{*} \leq 2 h / \sqrt{3}$. In Figures 20 to 22 , we represent the variations of $G_{r}^{N}$ along $D_{\pi / 6}$ for $t=3,5,8$. This time, the functions are singular for $r^{*}=c t$. However, one observes that the region where $G_{r}^{N}$ takes very large values becomes more and more confined close to the point $r^{*}=c t$ as $N$ increases.

For $\theta=\pi / 3, r^{*} \leq 2 h$. In Figures 23 to 25 , we represent the variations of $G_{r}^{N}$ along $D_{\pi / 3}$ for $t=3,5,8$. The functions are still singular for $r^{*}=c t$. The shape of the reflected wave is more complicated than for $\theta=\pi / 6$, especially for large $N$.

Angular variation of the reflected wave. Our previous results have already illustrated the dependence of the reflected field with respect to $\theta(x)$. Here, let us consider the relative error $\gamma_{r}^{N}(x, t)$ along the "reflected" wave front defined as 


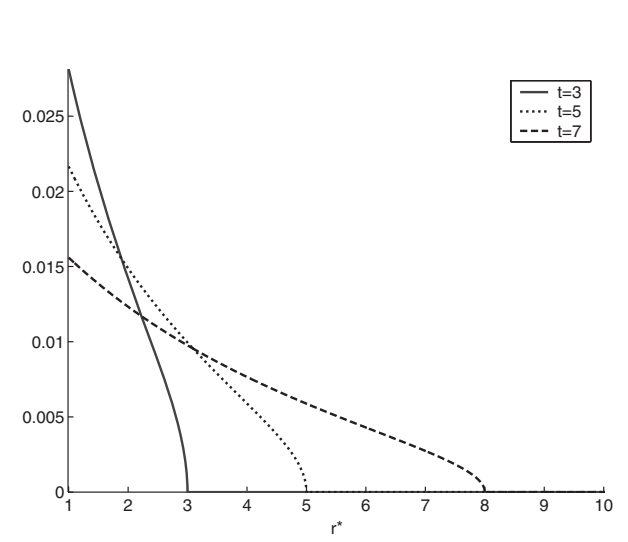

FIG. 17. $r^{*} \mapsto G_{r}^{1}\left(r^{*}, \theta=0, t\right)$.

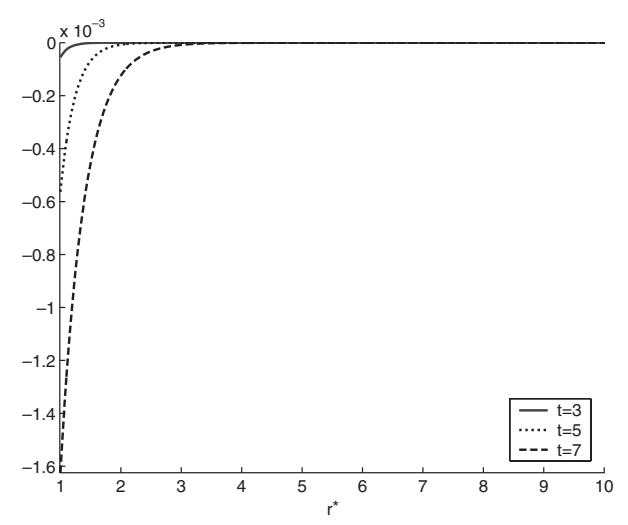

FIG. 19. $r^{*} \mapsto G_{r}^{10}\left(r^{*}, \theta=0, t\right)$.

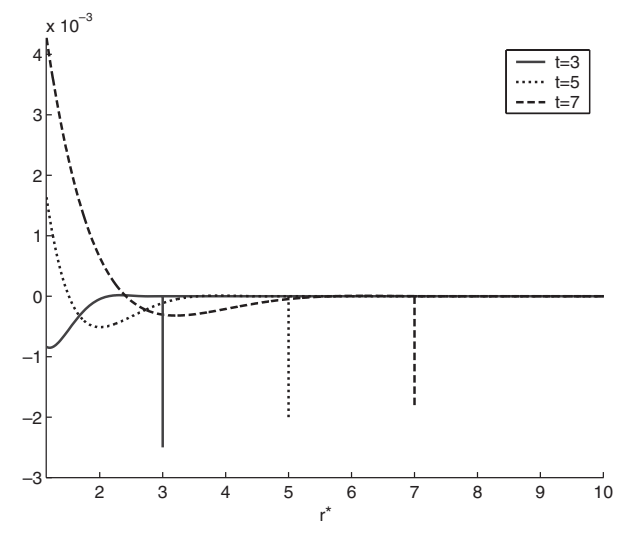

FIG. 21. $r^{*} \mapsto G_{r}^{5}\left(r^{*}, \theta=\pi / 6, t\right)$.

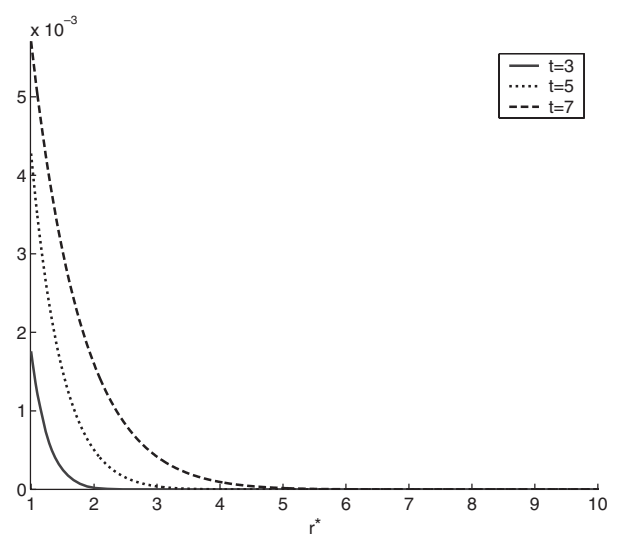

FIG. 18. $r^{*} \mapsto G_{r}^{5}\left(r^{*}, \theta=0, t\right)$.

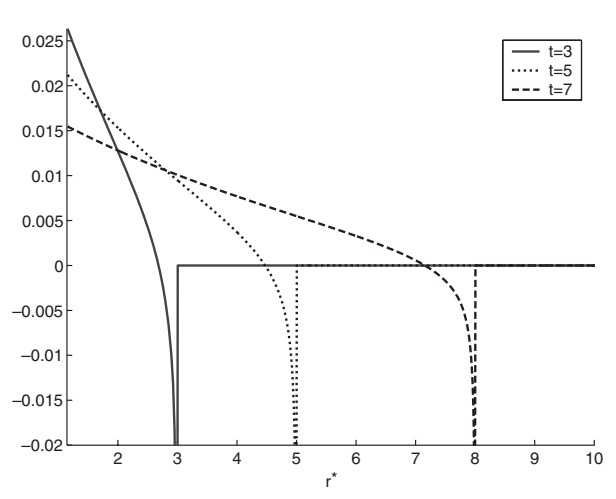

FIG. 20. $r^{*} \mapsto G_{r}^{1}\left(r^{*}, \theta=\pi / 6, t\right)$.

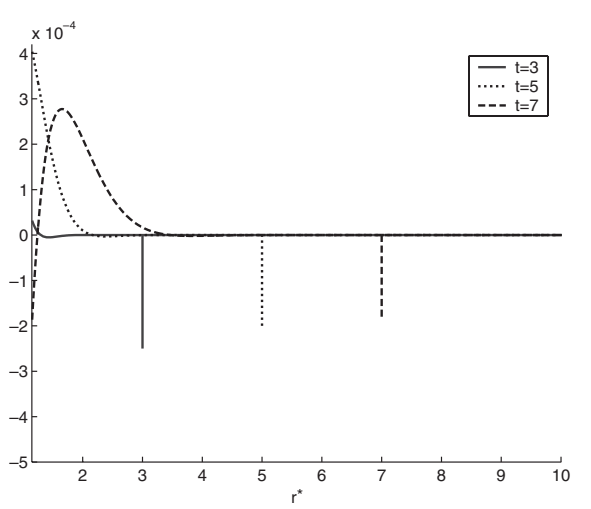

FIG. 22. $r^{*} \mapsto G_{r}^{10}\left(r^{*}, \theta=\pi / 6, t\right)$. 


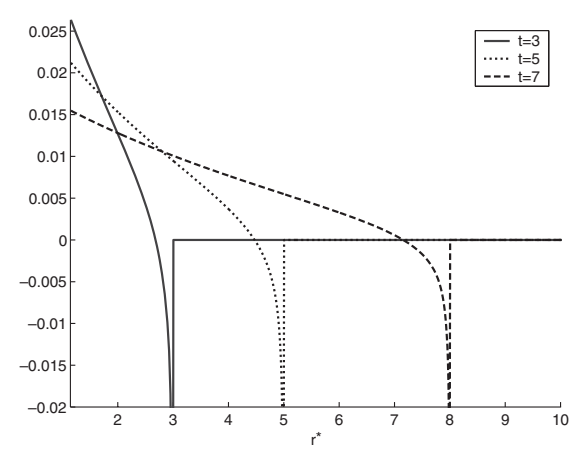

FIG. 23. $r^{*} \mapsto G_{r}^{1}\left(r^{*}, \theta=\pi / 3, t\right)$.



FIG. 24. $r^{*} \mapsto G_{r}^{5}\left(r^{*}, \theta=\pi / 3, t\right)$.

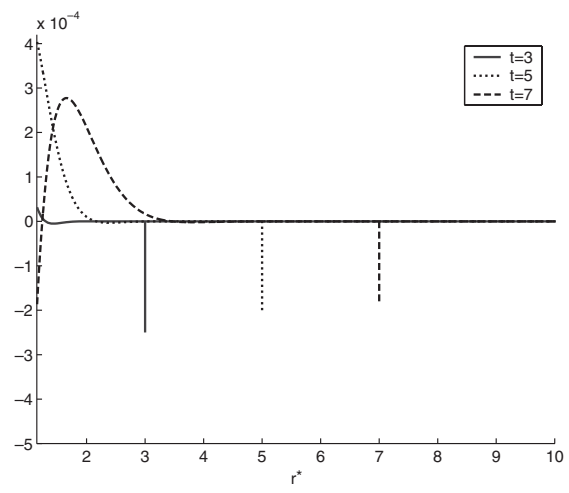

FIG. 25. $r^{*} \mapsto G_{r}^{10}\left(r^{*}, \theta=\pi / 3, t\right)$.

$$
W F_{r}(t)=\partial \Omega(t)=\left\{x \in \mathbb{R}_{+}^{2} / r^{*}(x)=c t\right\} \quad\left(\neq \emptyset \text { for } t>\frac{h}{c}\right) .
$$

Let $M_{\theta}(t)=(c t \sin \theta, c t \cos \theta) \in W F_{r}(t)$ (note that $M_{\theta}(t)$ describes $W F_{r}(t)$ when $\theta$ varies from $-\arccos \frac{h}{c t}$ to $\left.+\arccos \frac{h}{c t}\right)$; one easily deduces from (2.10) that

$$
\lim _{x \rightarrow M_{\theta}(t), x \in \Omega(t)} \gamma_{r}^{N}(x, t)=\mathbf{R}_{N}(\theta)=(-1)^{N}\left(\frac{1-\cos \theta}{1-\cos \theta}\right)^{N} .
$$

In other words, the curve representing, as a function of the direction $\theta$, the variations of the relative error $\gamma_{r}^{N}(x, t)$ along the "reflected" wave front $W F_{r}(t)$ is nothing but the portion of the curve of Figure 1 that corresponds to $-\arccos \frac{h}{c t} \leq \theta \leq \arccos \frac{h}{c t}$.

\subsection{The case of a source term.}

Comparison with numerical experiments. We have implemented a MATLAB code for the computation of the convolution integral (4.2). To validate our "exact" solution(!), we have compared our results with those obtained with a finite difference code written by F. Collino. In our experiment, the source function is a truncated first derivative of a Gaussian:

$$
f(t)=\frac{d}{d t}\left\{e^{-2 \pi f_{0}\left(t-t_{0}\right)^{2}}\right\} H\left(2 t_{0}-t\right), \quad f_{0}=10, t_{0}=1 / f_{0} .
$$



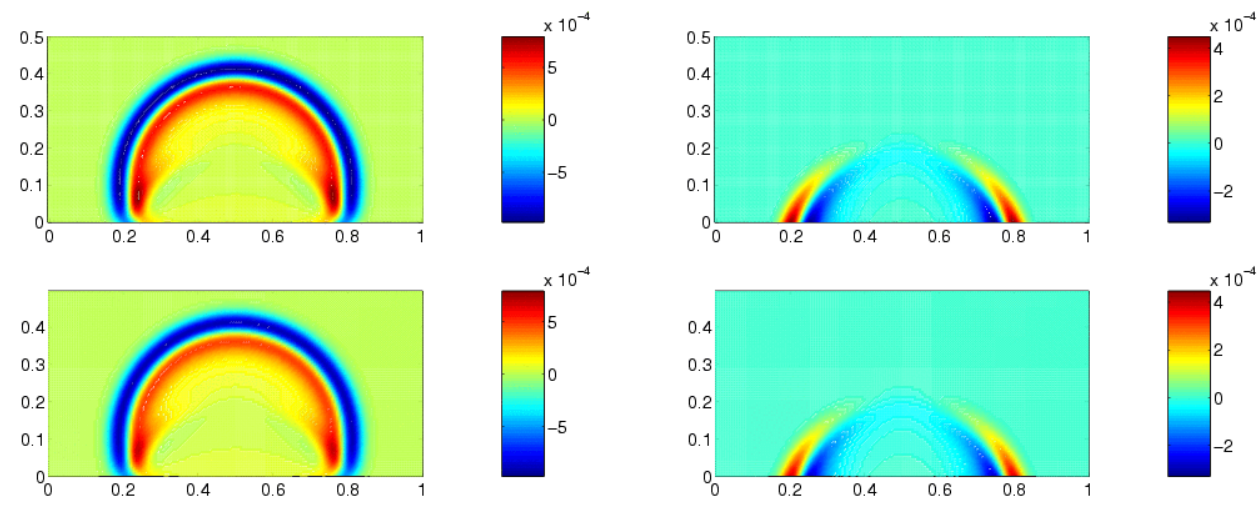

FIG. 26. Total field. $N=1$.

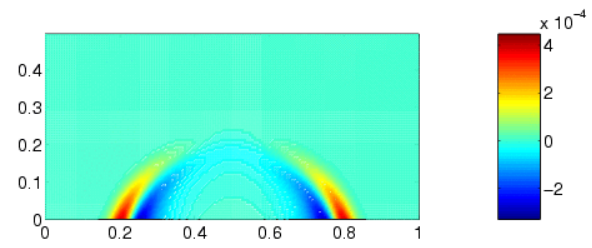

FIG. 27. Reflected field. $N=1$.
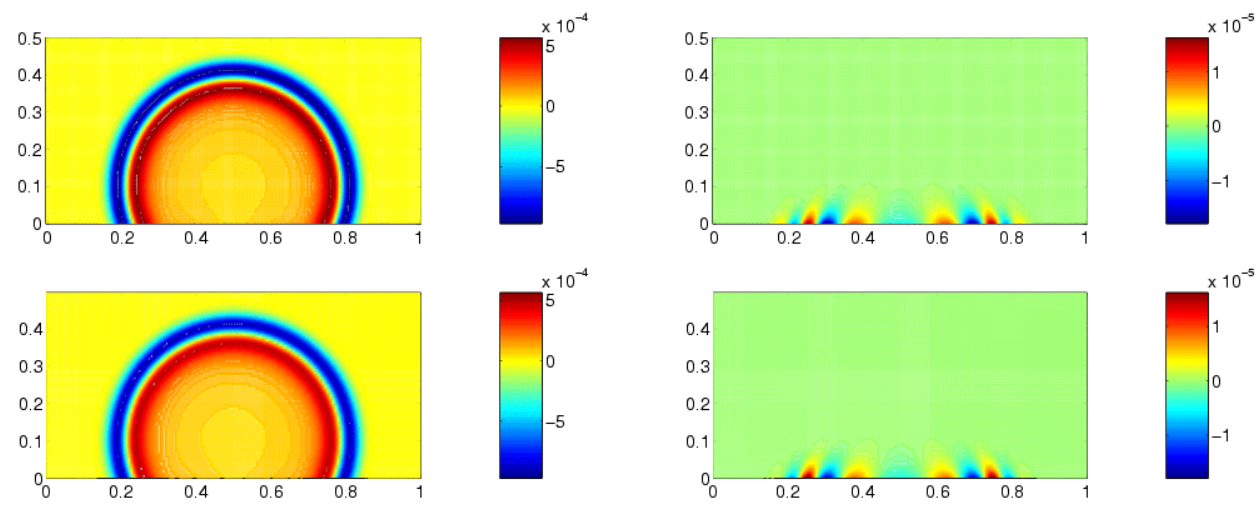

FIG. 28. Total field. $N=5$.

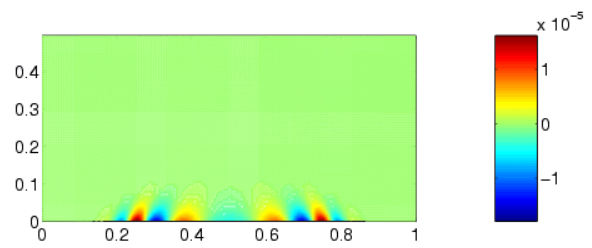

FIG. 29. Reflected field. $N=5$.

In Figures 26 to 29 we have compared the "analytical" solution (top picture in each panel) to the numerical one (bottom picture in each panel) for two values of $N: N=1$ and 5 . In each picture we represent the level lines of the solution at time $t=0.4$. The left pictures represent the total field while the right pictures represent the reflected field (the error). In each case, for the representation, the reflected field has been amplified by a factor which depends on $N: 1.3$ for $N=1$ and 25 for $N=5$. In each case, the results reveal a very good agreement between the two solutions. In Figures 30 to 33 we have compared both solutions at point $(0.9,0.1)$ as functions of time. The solid curves represent the "analytical" solution and the dashed curves the numerical one for two values of $N: N=1$ and 5 . As before the left pictures represent the total field while the right pictures represent the reflected field.

$L^{\infty}$ error estimates. In Figures 34 and 35 we have compared the $L_{\infty}$-norm of the reflected field (the solid curves) to the uniform estimates (2.15) and (2.16) given by Theorem 2.4 (the dashed curves) for $N=1,2,5,10$. The source is a step function in time: $f(t)=1$ if $0 \leq t \leq 2$ and $f(t)=0$ otherwise. Our estimate appears to be very sharp for $N=1$ and becomes less accurate (although quite acceptable) as $N$ increases. Since we used the $L^{\infty}$-norm of the source function to establish our error estimates, one could imagine that this estimate is not very sharp for more complicated source 


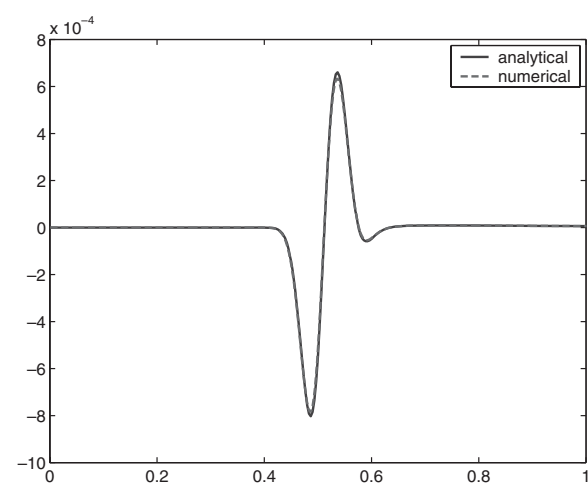

FIG. 30. Total field. $N=1$.

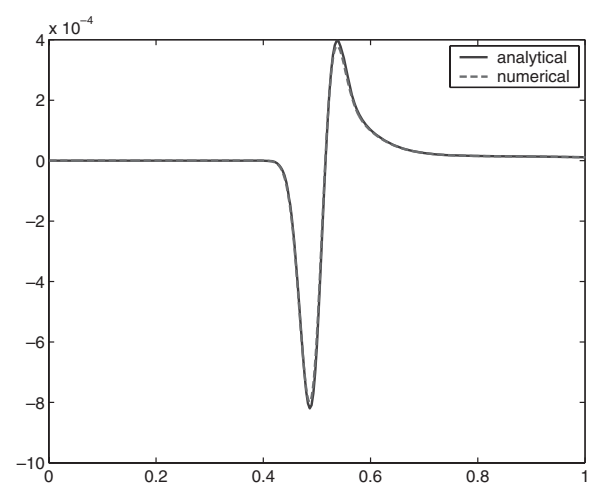

FIG. 32. Total field. $N=5$.

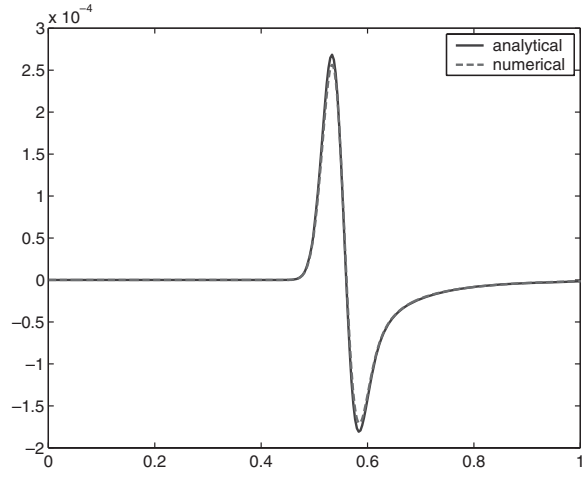

FIG. 31. Reflected field. $N=1$.

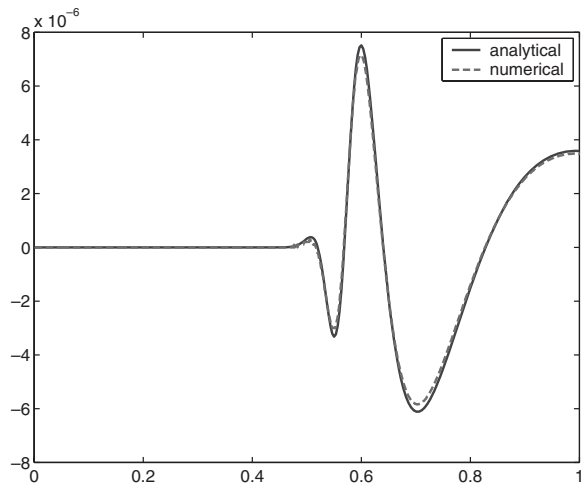

FIG. 33. Reflected field. $N=5$.

functions. To check this, we have repeated the previous experiment when the source is still given by (5.7) with $f_{0}=1$. Figures 36 and 37 illustrate these experiments for $N=1$ and 5 . The estimate is obviously less accurate than in the case of the step source function but still gives a reasonable bound.

6. Conclusion and perspectives. The use of the Cagniard-De Hoop method has enabled us to obtain a quasi-analytical representation of the field reflected by Engquist-Majda higher order ABCs. This permits us to obtain new theoretical estimates for the time-dependent problem.

Of course, the method can be applied to other boundary conditions (we give in the appendix the example of Higdon's boundary conditions). It would also be interesting to treat other equations such as Maxwell's equations or elastodynamics equations. One also might think that the Cagniard-De Hoop method could be a new tool for analyzing the stability of boundary conditions.

In a forthcoming work, we wish to treat the case of the PMLs for ABCs. This should give some insights about the quantitative comparison between $\mathrm{ABCs}$ and PMLs. 


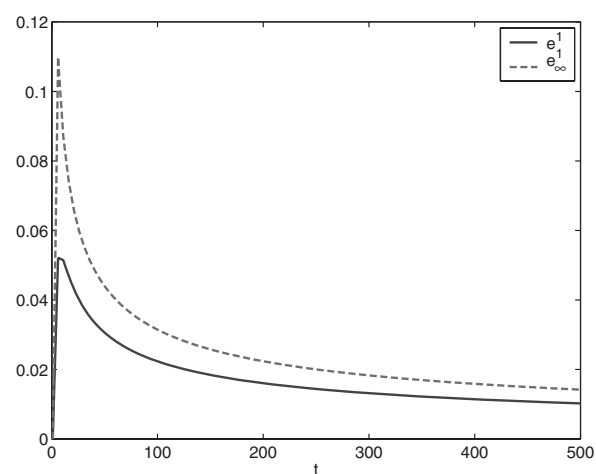

FIG. 34. Error estimates. $N=1$.

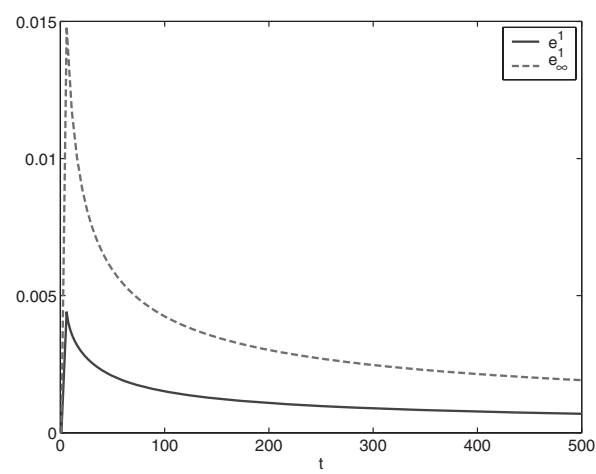

FIG. 36. Error estimates. $N=1$.

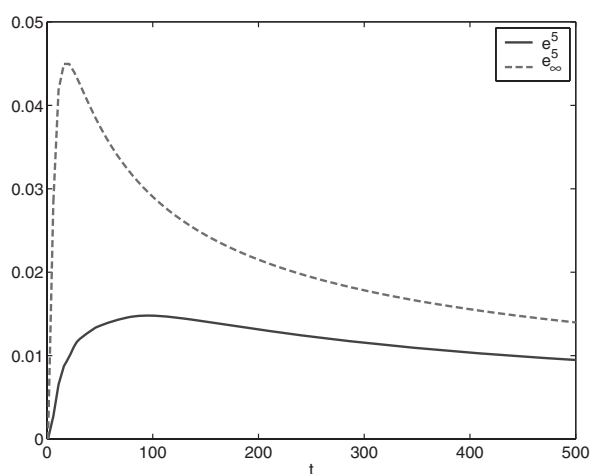

FIG. 35. Error estimates. $N=5$.

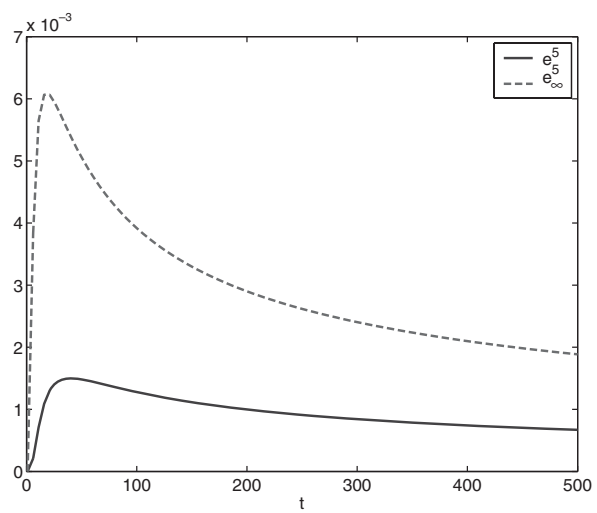

FIG. 37. Error estimates. $N=5$.

Appendix. Extension to Higdon's boundary conditions. In 1986 Higdon [23], [21] proposed another approximation of the condition (1.2) of the form

$$
B_{H i g}^{N} u=\prod_{j=1}^{N}\left(\cos \alpha_{j} \frac{\partial}{\partial t}-c \frac{\partial}{\partial x}\right) u=0 .
$$

These conditions are a generalization of the condition (1.5) (obtained with $\alpha_{j}=0$ for all $j$ ) and have the property to be exactly satisfied by any linear combination of plane waves whose angle of incidence is $\alpha_{j}$.

Using the same method as in section 3 , it can be shown that the solution of the problem

$$
\begin{cases}\text { Find } u: \mathbb{R}_{+}^{2} \times \mathbb{R} \mapsto \mathbb{R} & \text { such that } \\ \frac{1}{c^{2}} \frac{\partial^{2} u}{\partial t^{2}}-\Delta u=\delta\left(x-x_{S}\right) \times \delta(t) & \text { in } \mathbb{R}_{+}^{2}, \\ \mathcal{B}_{H i g}^{N} u=0 & \text { on } \Gamma, \\ u(x, t)=0 & \text { for } t<0\end{cases}
$$


is given by

$$
u(x, t)=G_{H i g}^{N}(x, t)=G_{i}(x, t)+G_{H i g, r}^{N}(x, t),
$$

where

(A.4)

$$
\mid \begin{aligned}
& G_{i}(x, t)=\frac{1}{2 \pi \sqrt{t^{2}-\frac{r(x)^{2}}{c^{2}}}} H(c t-r(x)), \\
& G_{H i g, r}^{N}(x, t)=\frac{1}{2 \pi \sqrt{t^{2}-\frac{r^{*}}{c^{2}}}}\left[\prod_{j=1}^{n} \rho_{j}(x, t)\right] \cos \left[\sum_{j=1}^{n} \psi_{i}(x, t)\right] H\left(c t-r^{*}(x)\right),
\end{aligned}
$$

where $\rho_{j}(x, t)$ and $\psi_{i}(x, t)$ are given by

$$
\rho_{j}(x, t)=\sqrt{\frac{\left(c t-a_{j}\right)^{2}-b_{j}^{2}}{\left(c t+a_{j}\right)^{2}-b_{j}^{2}}}
$$

and

$$
\begin{aligned}
& \psi_{j}(x, t)=\arccos \left[\frac{r^{*}(x, t)^{2}-c^{2} t^{2}+r^{*}(x, t)^{2} \cos ^{2} \alpha_{j}-r^{*}(x, t)^{2} \cos ^{2} \theta}{\sqrt{\left(\left(c t-a_{j}\right)^{2}-b_{j}^{2}\right)\left(\left(c t+a_{j}\right)^{2}-b_{j}^{2}\right)}}\right], \\
& \psi_{j}(x, t)=\arccos \left[\frac{r^{*}(x, t)^{2}-c^{2} t^{2}+r^{*}(x, t)^{2} \cos ^{2} \alpha_{j}-\left(x_{2}+h\right)^{2}}{\sqrt{\left(\left(c t-a_{j}\right)^{2}-b_{j}^{2}\right)\left(\left(c t+a_{j}\right)^{2}-b_{j}^{2}\right)}}\right]
\end{aligned}
$$

with

$$
a_{j}=r^{*}(x) \cos \alpha_{j} \cos \theta=\cos \alpha_{j}\left(x_{2}+h\right)
$$

and

$$
b_{j}=r^{*}(x) \sin \theta \sin \alpha_{j}=x_{1} \sin \alpha_{j} .
$$

Thanks to (A.5), one can see that the function $x \mapsto G_{H i g, r}^{N}(x, t)$ is singular on the circle $r^{*}(x)=$ ct except in the directions $\alpha_{j}$.

\section{REFERENCES}

[1] B. Alpert, L. Greengard, and T. Hagstrom, Rapid evaluation of nonreflecting boundary kernels for time-domain wave propagation, SIAM J. Numer. Anal., 37 (2000), pp. 11381164.

[2] B. Alpert, L. Greengard, and T. Hagström, Nonreflecting boundary conditions for the time-dependent wave equation, J. Comput. Phys., 180 (2002), pp. 270-296.

[3] G. Baker, Essentials of Padé Approximants, Academic Press, New York, 1975.

[4] H. BARUCQ, A new family of first-order boundary conditions for the Maxwell system: Derivation, well-posedness and long-time behavior, J. Math. Pures Appl., 9 (2003), pp. 67-88.

[5] J. BÉRENGER, Three-dimensional perfectly matched layer for the absorption of electromagnetic waves, J. Comput. Phys., 127 (1996), pp. 363-379.

[6] J. P. BÉREnger, A perfectly matched layer for the absorption of electromagnetic waves, J. Comput. Phys., 114 (1994), pp. 185-200.

[7] L. Cagniard, Réflexion et Réfraction des Ondes Sismiques Progressives, Gauthier-Villars, Paris, 1939 
[8] T. H. DuOng And P. Joly, On the stability analysis of boundary conditions for the wave equation by energy methods. Part I: The homogeneous case, Math. Comp., 62 (1994), pp. 539-563.

[9] B. Engquist and L. Halpern, Far field boundary conditions for computation over long time, Appl. Numer. Math., 4 (1988), pp. 21-45.

[10] B. EngQuist AND L. HALPERn, Long-time behaviour of absorbing boundary conditions, Math. Methods Appl. Sci., 13 (1990), pp. 189-203.

[11] B. EngQuist and A. MAJda, Absorbing boundary conditions for the numerical simulation of waves, Math. Comp., 31 (1977), pp. 629-651.

[12] B. Engquist And A. Majda, Radiation boundary conditions for acoustic and elastic wave calculations, Comm. Pure Appl. Math., 32 (1979), pp. 314-358.

[13] D. Givoli, Exact and high order non-reflecting computational boundaries, in Mathematical and Numerical Aspects of Wave Propagation, G. Cohen, E. Heikkola, P. Joly, and P. Neittanmaki, eds., Springer, Berlin, 2003, pp. 26-31.

[14] M. J. Grote And J. B. Keller, Exact nonreflecting boundary conditions for the time dependent wave equation, SIAM J. Appl. Math., 55 (1995), pp. 280-297.

[15] M. J. Grote And J. B. Keller, Nonreflecting boundary conditions for time dependent scattering, J. Comput. Phys., 127 (1996), pp. 52-81.

[16] T. HaGström, On the convergence of local approximations to pseudodifferentials operators, in Proceedings of the 3rd International Conference on Mathematical and Numerical Aspects of Wave Propagation, E. Bécache, G. Cohen, P. Joly, and J. Roberts, eds., SIAM, Philadelphia, 1995, pp. 474-482.

[17] T. Hagström, On high-order radiation boundary conditions, in Computational Wave Propagation (Minneapolis, MN, 1994/1995), B. Engquist and G. A. Kriegsmann, eds., IMA Vol. Math. Appl. 86, Springer, New York, 1997, pp. 1-21.

[18] T. Hagström, Radiation boundary conditions for the numerical simulation of waves, Acta Numer., 8 (1999), pp. 47-106.

[19] T. Hagström, New results on absorbing layers and radiation boundary conditions, in Topics in Computational Wave Propagation: Direct and Inverse Problems, M. Ainsworth, P. Davies, D. Duncan, P. Martin, and B. Rynne, eds., Lect. Notes Comput. Sci. Eng. 31, Springer, Berlin, 2003, pp. 1-42.

[20] L. Halpern and J. Rauch, Error analysis for absorbing boundary conditions, Numer. Math., 51 (1987), pp. 459-467.

[21] R. L. Higdon, Absorbing boundary conditions for difference approximations to the multidimensional wave equation, Math. Comp., 47 (1986), pp. 437-459.

[22] R. L. Higdon, Initial-boundary value problems for linear hyperbolic systems, SIAM Rev., 28 (1986), pp. 177-217.

[23] R. L. Higdon, Numerical absorbing boundary conditions for the wave equation, Math. Comp., 4 (1987), pp. 65-90.

[24] J. V. D. HiJden, Propagation of Transient Elastic Waves in Stratified Anisotropic Media, North-Holland Ser. Appl. Math. Mech. 32, North-Holland, Amsterdam, 1987.

[25] A. T. D. Hoop, The surface line source problem, Appl. Sci. Res. B, 8 (1959), pp. 349-356.

[26] H. O. Kreiss, Initial boundary value problems for hyperbolic systems, Comm. Pure Appl. Math., 23 (1970), pp. 277-298.

[27] L. N. TRefethen And L. HalPeRn, Well-posedness of one-way wave equations and absorbing boundary conditions, Math. Comp., 47 (1986), pp. 421-435. 